\title{
GMPPA defects cause a neuromuscular disorder with $\alpha$-dystroglycan hyperglycosylation
}

\author{
Patricia Franzka, ${ }^{1}$ Henriette Henze, ${ }^{2}$ M. Juliane Jung, ${ }^{2}$ Svenja Caren Schüler, ${ }^{2}$ Sonnhild Mittag, ${ }^{3}$ Karina Biskup, ${ }^{4}$ Lutz Liebmann, ${ }^{1}$ \\ Takfarinas Kentache, ${ }^{5}$ José Morales, ${ }^{6}$ Braulio Martínez, ${ }^{7}$ Istvan Katona, ${ }^{8}$ Tanja Herrmann, ${ }^{1}$ Antje-Kathrin Huebner, ${ }^{1}$ \\ J. Christopher Hennings, ${ }^{1}$ Susann Groth, ${ }^{2}$ Lennart Gresing, ${ }^{1}$ Rüdiger Horstkorte, ${ }^{9}$ Thorsten Marquardt, ${ }^{10}$ Joachim Weis, ${ }^{8}$ \\ Christoph Kaether, ${ }^{2}$ Osvaldo M. Mutchinick, ${ }^{6}$ Alessandro Ori, ${ }^{2}$ Otmar Huber, ${ }^{3}$ Véronique Blanchard, ${ }^{4}$ Julia von Maltzahn, ${ }^{2}$ \\ and Christian A. Hübner ${ }^{1}$
}

\begin{abstract}
IInstitute of Human Genetics, University Hospital Jena, Friedrich Schiller University, Jena, Germany. ${ }^{2}$ Leibniz-Institute on Aging - Fritz-Lipmann-Institute, Jena, Germany. ${ }^{3}$ Department of Biochemistry II, University Hospital Jena, Friedrich Schiller University, Jena, Germany. ${ }^{4}$ Charité-Universitätsmedizin Berlin, corporate member of Freie Universität Berlin, Humboldt-Universität zu Berlin, and Berlin Institute of Health, Institute of Laboratory Medicine, Clinical Chemistry and Pathobiochemistry, Berlin, Germany. ${ }^{5}$ Welbio and de Duve Institute, Université Catholique de Louvain, Brussels, Belgium. ${ }^{6}$ Department of Cenetics, Instituto Nacional de Ciencias Médicas y Nutrición Salvador Zubirán, Mexico City, Mexico. ${ }^{7}$ Department of Pathology, Instituto Nacional de Ciencias Médicas y Nutrición Salvador Zubirán, Mexico City, Mexico. ${ }^{8}$ Institut für Neuropathologie, Institute of Neuropathology, RWTH Aachen University Hospital, Aachen, Cermany. ${ }^{9}$ Institut für Physiologische Chemie, Martin-Luther-Universität HalleWittenberg, Halle, Germany. ${ }^{10}$ University Hospital Muenster, Department of Pediatrics, Muenster, Germany.
\end{abstract}

GDP-mannose-pyrophosphorylase-B (CMPPB) facilitates the generation of CDP-mannose, a sugar donor required for glycosylation. GMPPB defects cause muscle disease due to hypoglycosylation of $\alpha$-dystroglycan ( $\alpha$-DG). AlphaDC is part of a protein complex, which links the extracellular matrix with the cytoskeleton, thus stabilizing myofibers. Mutations of the catalytically inactive homolog GMPPA cause alacrima, achalasia, and mental retardation syndrome (AAMR syndrome), which also involves muscle weakness. Here, we showed that Gmppa-KO mice recapitulated cognitive and motor deficits. As structural correlates, we found cortical layering defects, progressive neuron loss, and myopathic alterations. Increased CDP-mannose levels in skeletal muscle and in vitro assays identified GMPPA as an allosteric feedback inhibitor of GMPPB. Thus, its disruption enhanced mannose incorporation into glycoproteins, including $\alpha$-DC in mice and humans. This increased $\alpha$-DG turnover and thereby lowered $\alpha$-DG abundance. In mice, dietary mannose restriction beginning after weaning corrected $\alpha$-DG hyperglycosylation and abundance, normalized skeletal muscle morphology, and prevented neuron degeneration and the development of motor deficits. Cortical layering and cognitive performance, however, were not improved. We thus identified GMPPA defects as the first congenital disorder of glycosylation characterized by $\alpha$-DC hyperglycosylation, to our knowledge, and we have unraveled underlying disease mechanisms and identified potential dietary treatment options.

\section{Introduction}

Cell surface proteins are important for the attachment of a cell to the extracellular matrix (ECM). They are modified by posttranslational covalent attachment of branched carbohydrate oligomers. $N$-linked oligosaccharides are linked to asparagine; $O$-linked glycans are mainly attached to serine, threonine, or tyrosine. The process of glycosylation starts at the ER and proceeds in the Golgi apparatus. Abnormal glycosylation of proteins can induce deleterious effects, as observed in congenital disorders of glycosylation, which often result in serious, sometimes fatal malfunctions of different organ systems such as the brain and muscle (1). One example is mutations in the gene encoding GDP-mannose-pyrophosphorylase-B (GMPPB), which result in

Authorship note: $\mathrm{HH}$ and JJ contributed equally to this work Conflict of interest: The authors have declared that no conflict of interest exists. Copyright: @ 2021, American Society for Clinical Investigation. Submitted: April 17, 2020; Accepted: March 18, 2021; Published: May 3, 2021. Reference information: J Clin Invest. 2021;131(9):e139076. https://doi.org/10.1172/JCl139076. variable skeletal muscle disorders, including muscular dystrophy and myasthenia (2-5), which are characterized by hypoglycosylation of the sarcolemma-associated protein $\alpha$-dystroglycan $(\alpha-D G)$. GMPPB catalyzes the production of GDP-mannose from mannose-1-phosphate and GTP (6), which is required as a sugar donor for glycosylation of proteins. We recently identified mutations in the gene encoding GDP-mannose-pyrophosphorylase-A (GMPPA) in patients with a clinically variable syndrome characterized by symptoms such as muscle weakness, muscular hypotonia, gait abnormalities, problems swallowing (achalasia), reduced or absent tear flow (alacrima), and mental retardation, which is also known as AAMR syndrome (7). Whether GMPPA plays a role in the glycosylation of $\alpha$-DG remains unclear because GMPPA is catalytically inactive (6).

Alpha-DG is a highly glycosylated extracellular peripheral membrane protein noncovalently attached to the transmembrane protein $\beta$-dystroglycan ( $\beta$-DG). Both proteins are encoded by a single gene (DAG1) and are generated by cleavage of a common precursor. Alpha-DG binds to ECM components such as laminin via its glycan side chains, and $\beta$-DG is connected to cytoplasmic pro- 
teins including dystrophin. These proteins are part of the dystroglycan complex (DGC), which acts as a linker between the ECM and the intracellular cytoskeleton, thereby stabilizing myofibers (8, 9). Mutations in different members of the DGC lead to muscular dystrophy in mice and humans and affect myofibers and muscle stem cells (10). Glycosylation is the crucial modification that modulates the function of $\alpha-D G$ as a receptor for extracellular binding partners. Accordingly, hypoglycosylation of $\alpha-D G$ is a central event in the pathogenesis of several complex muscle disorders, such as Walker-Warburg syndrome, which also affects the brain (11).

We showed that binding of GMPPA to GMPPB inhibited the activity of recombinant GMPPB in a GDP-mannose dependent manner, suggesting that GMPPA acts as an allosteric feedback inhibitor of GMPPB. Gmppa-KO mice showed cognitive and motor impairment with progressive neuron loss and myopathic alterations. In agreement with a role as an allosteric inhibitor of GMPPB, GDP-mannose levels were increased in skeletal muscle of Gmppa-KO mice and led to $\alpha$-DG hyperglycosylation. Knockdown studies in myoblasts further revealed that the turnover of hyperglycosylated $\alpha-D G$ was increased, thus leading to a reduced $\alpha-D G$ abundance. A myopathic disorder characterized by $\alpha$-DG hyperglycosylation and decreased overall $\alpha$-DG abundance was also evident in skeletal muscle biopsies of patients with AAMR. In mice, $\alpha$-DG hyperglycosylation, neuron loss, muscle damage, and motor deficits could be prevented by a mannose-free diet starting at P14.

\section{Results}

Gmppa-KO mice show progressive motor deficits and cognitive impairments. To study GMPPA deficiency, we generated a GmppaKO mouse model from targeted embryonic stem cell clones obtained from EUCOMM (EPD0621_7_G03) (Figure 1A). Chimeric mice were verified by Southern blot (Supplemental Figure 1A; supplemental material available online with this article; https:// doi.org/10.1172/JCI139076DS1) and subsequently mated with FLPe- (12) and Cre-deleter mice (13). Mating of offspring with a heterozygous deletion of exon 5 resulted in the generation of homozygous mice at the expected Mendelian ratio. The predicted aberrant transcript devoid of exon 5 is predicted to lead to a frameshift and a premature termination of protein translation. Gmppa transcript abundance was drastically decreased in $\mathrm{KO}$ mice, suggesting that the aberrant transcript undergoes nonsense-mediated decay (Supplemental Figure 1B). Immunoblot analysis for GMPPA detected bands of the expected size in different tissues from WT mice, which were absent in lysates of mice with a homozygous deletion of exon 5 (Figure 1B), further validating the successful knockout of GMPPA.

The bodyweight of young Gmppa-KO mice (3 months of age) was indistinguishable from WT littermates, whereas a significant decrease was observed at 12 months of age (Supplemental Figure $1, \mathrm{C}$ and D). To address whether KO mice develop gait abnormalities, as reported for many patients with AAMR (7), we performed a beam-walk test (14). The foot-base angle of the hind paw at toe-off position was reduced in $\mathrm{KO}$ mice at 3 months of age and further flattened over time (Figure 1C), suggesting a progressive muscle weakness. Progressive muscle weakness was also evident for the forelimbs in the weights test (Figure 1D). Moreover, aged KO mice fell off a wire mesh more rapidly than control littermates (Figure 1E).
To assess cognitive function, we performed a contextual and cued fear-conditioning test with 3- and 12-month-old WT and $\mathrm{KO}$ mice (Figure $1 \mathrm{~F}$ ), which tests the ability of KO mice to learn and remember an association between environmental cues and adverse experiences. Freezing behavior during the test was measured as an index of fear memory. For acquisition, mice were placed into a conditioning chamber and were given pairings of the conditioned auditory stimulus (the auditory cue) and an electric foot-shock. After 24 hours, mice were either exposed to a different chamber with presentation of the auditory cue (cued test) or the same context as for acquisition (context test). Although both WT and KO mice learned the conditioned stimulus, KO mice were unable to remember the context of the aversive stimulus, which suggests a defect in hippocampusdependent learning in Gmppa-KO mice (15). Taken together, Gmppa-KO mice reproduced cognitive and motor impairments as reported for patients with AAMR.

Gmppa disruption in mice affects brain development and long-term neuronal maintenance. The histological analysis of the somatosensory cortex revealed that the layering of the neocortex was altered in young Gmppa-KO mice (Figure 2A). Apart from this developmental defect, we also observed an age-dependent loss of hippocampal pyramidal neurons (Figure 2B) and Purkinje cells (Figure 2C).

We also analyzed the peripheral nervous system. Sciatic nerve fiber numbers at 12 months of age did not differ between genotypes and the distribution of axon diameters was normal (Figure 2D). In agreement, compound muscle action potentials at the tail tip in response to electrical stimulation at the root of the tail and the efferent nerve conduction velocity were not altered in 12-month-old KO mice (Figure 2E). Although the sensory amplitudes were unaffected, we noted a mild decrease of the sensory velocity in 12-month-old KO mice (Figure 2F). These data showed that loss of GMPPA entailed neurodevelopmental as well as neurodegenerative components, which mainly manifested in the central nervous system.

Gmppa-KO mice develop an age-dependent myopathy. Consistent with progressive motor impairments and decreased muscle strength, the mass of the M. tibialis anterior, the M. gastrocnemius together with M. soleus, and the M. palmaris longus was lower in 12-month-old KO mice (Figure 3A). Serum levels of creatinine kinase as a sign of pronounced muscle damage were not increased at 3 months (Supplemental Figure 2A) and 12 months of age (data not shown). A decrease of the mean myofiber diameter and an increased number of centrally located nuclei in musculus tibialis anterior sections was evident at 12 months of age (Figure 3B). A myofiber type-specific analysis is shown for the M. gastrocnemius/soleus in Supplemental Figure 6. Of note, we did not observe grouped degenerating myofibers as a typical sign of neurogenic muscle degeneration. TUNEL staining revealed a significant increase in apoptotic myofibers in KO samples (Figure 3C). Picro-Sirius red staining excluded a relevant fibrosis of skeletal muscles in Gmppa-KO mice, as often observed in muscular dystrophies (Figure 3D). We did not observe changes in the oxidative potential as judged from NADH-diaphorase staining (Supplemental Figure 2B). Staining for developmental myosin heavy chain, a marker for regenerating myofibers, suggested that muscle regeneration was increased (Figure $3 \mathrm{E}$ ). 
A

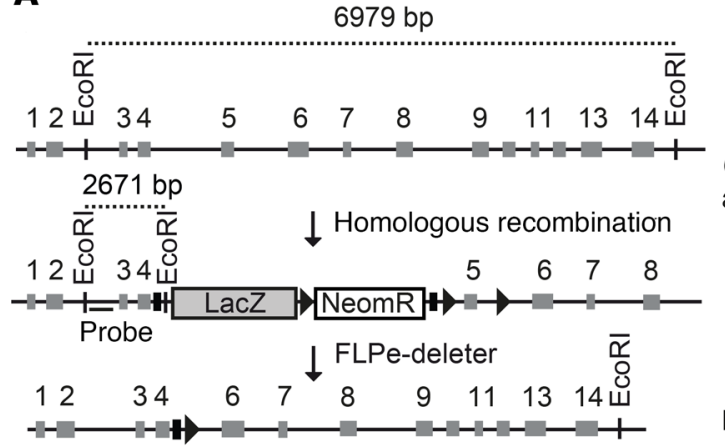

B

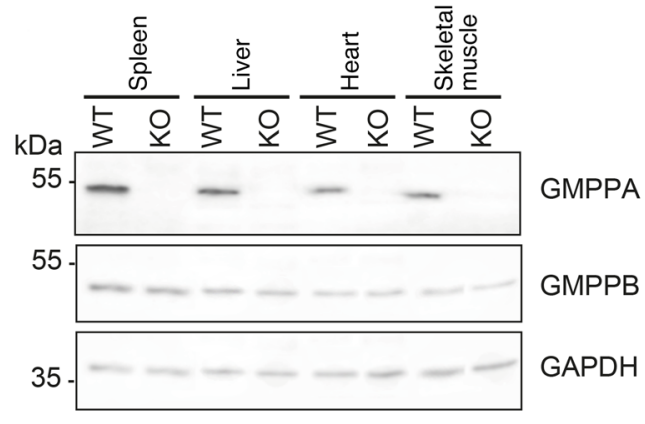

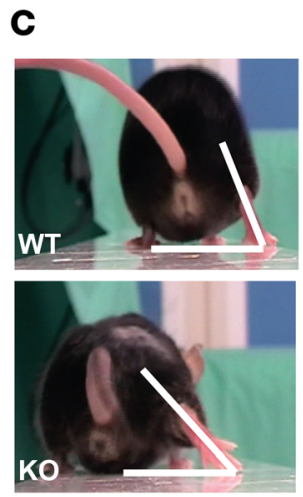

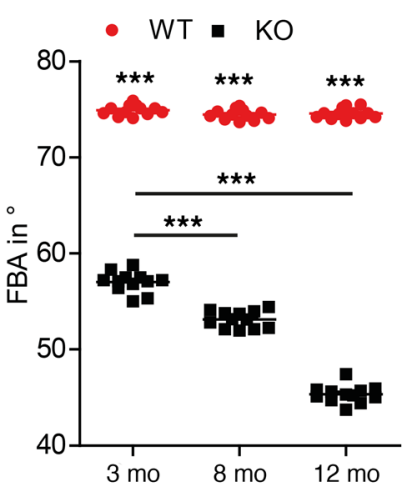

Gmppa WT allele

Recombinant allele

KO allele
E

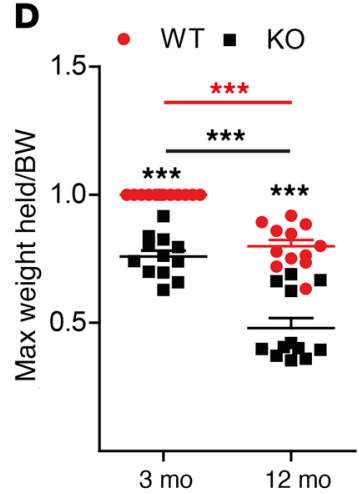

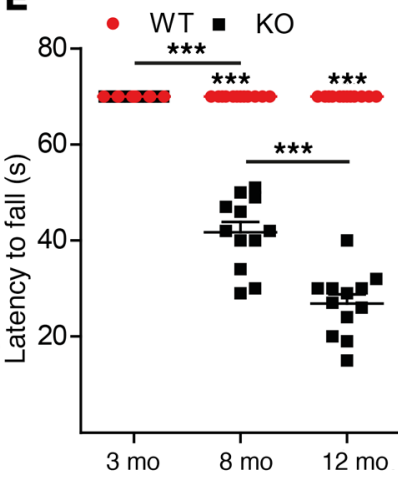

$\mathbf{F}$
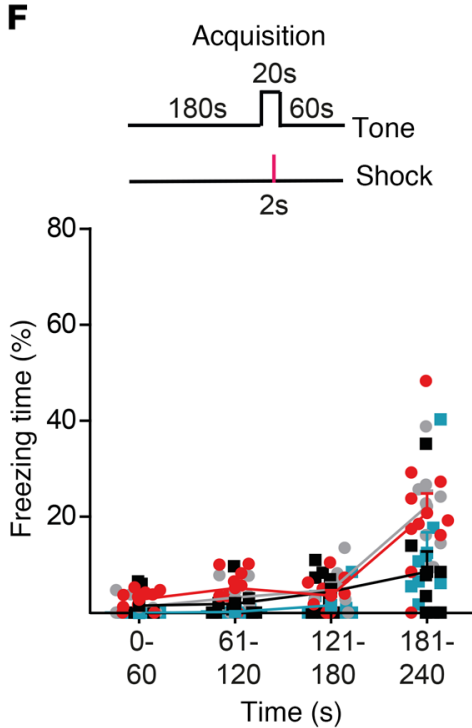

Cued test

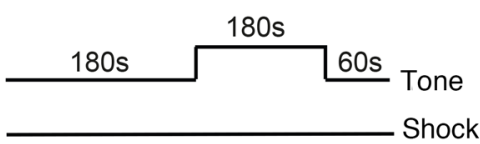

Context test
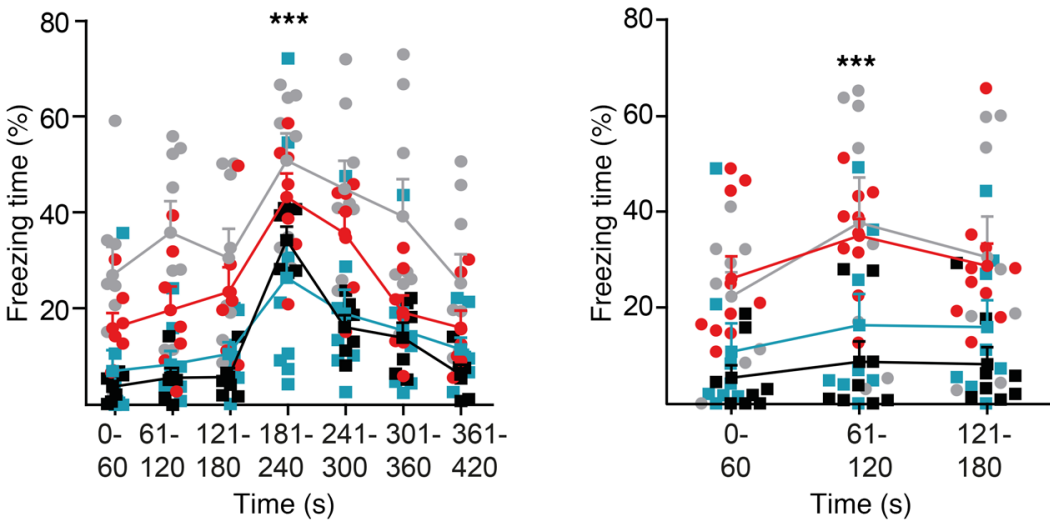
- WT 3 mo
- WT 12 mo
- $\mathrm{KO} 3 \mathrm{mo}$
- KO 12 mo

Figure 1. Gmppa-KO mice show progressive motor deficits and cognitive impairments. (A) Genomic structure of the WT and targeted Gmppa locus. Gray rectangles: exons; black rectangles: Frt sites; black triangles: loxP sites; LacZ: $\beta$-galactosidase cassette; NeomR: neomycin-resistance cassette. Black lines: probe-binding sites. (B) The GMPPA protein was detected in different tissues of WT mice, but was absent in KO samples. GMPPB abundance was not altered in Gmppa-KO mice. GAPDH served as loading control. (C) The foot-base angle (FBA) flattened in KO mice ( $n=12$ mice per group; 2-way ANOVA with Bonferroni post hoc test). (D) KO mice could hold less weight with their forepaws ( $n=12$ mice per group; 2-way ANOVA with Bonferroni post hoc test). (E) Aged KO mice fell off earlier from an inverted screen ( $n=12$ mice per group; 2-way ANOVA with Bonferroni post hoc test). (F) Cued and contextual fear conditioning test in 3- and 12-month-old WT and KO mice. Freezing behavior during the test was measured as an index of fear memory. For acquisition, mice were placed into a conditioning chamber and were given pairings of a tone and an electric foot-shock. After 24 hours, mice were either exposed to a different chamber with presentation of the auditory cue (cued test) or the same context as for acquisition (context test). Although both genotypes remembered the conditioned stimulus, KO mice were unable to remember the context of the aversive stimulus $(n=12$ mice per group; 2 -way ANOVA with Bonferroni post hoc test). Quantitative data are presented as mean \pm SEM with individual data points. ${ }^{* * *} P<0.0005$. 
A

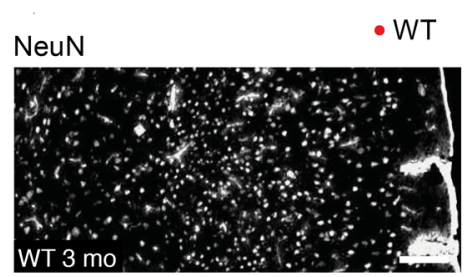

B

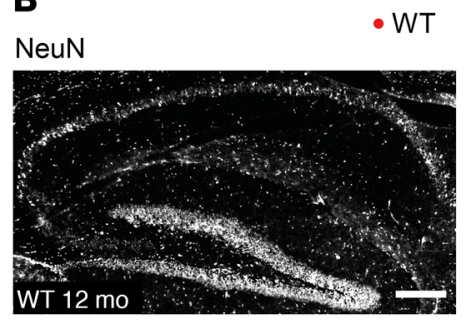

C

Calbindin
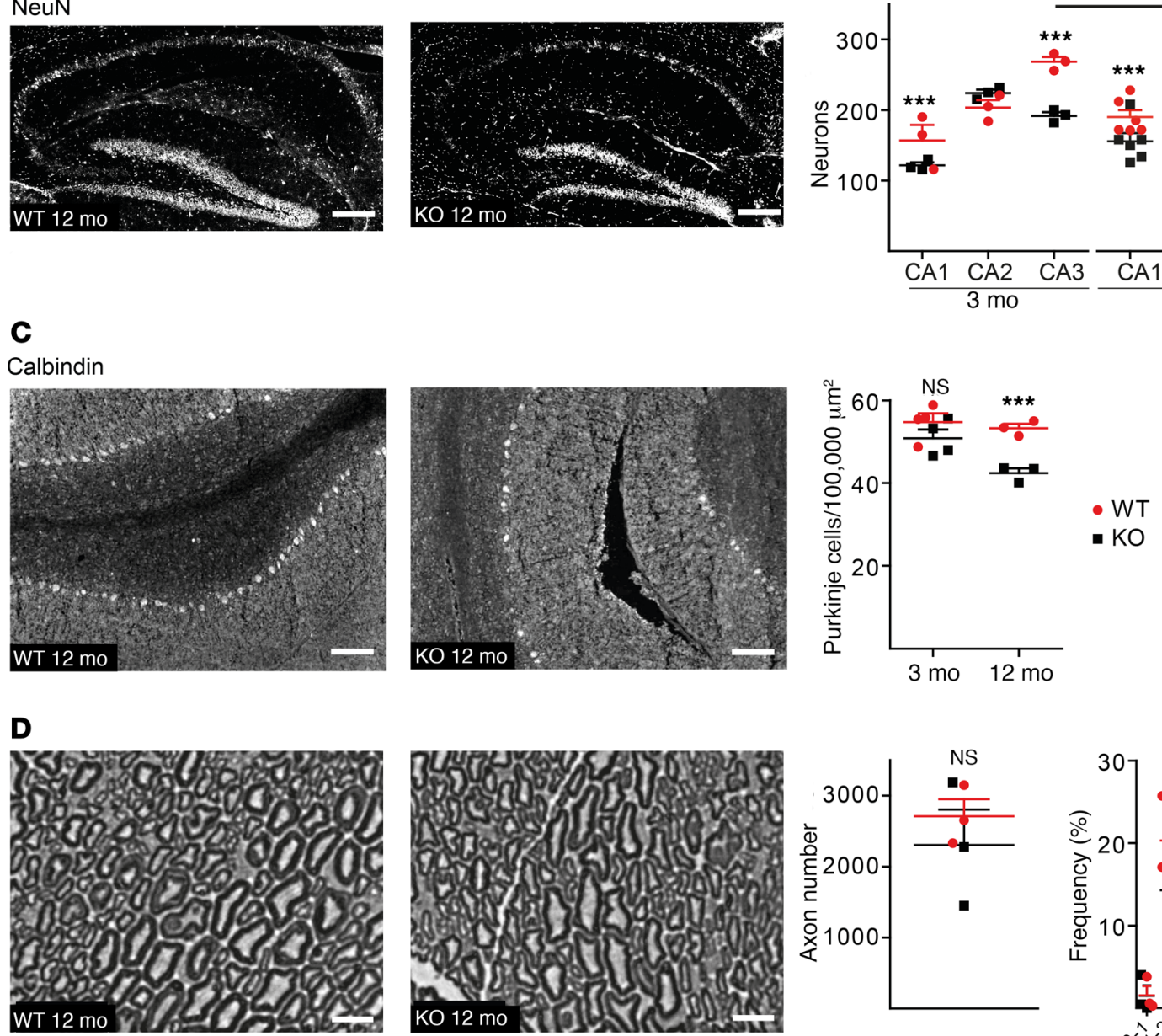

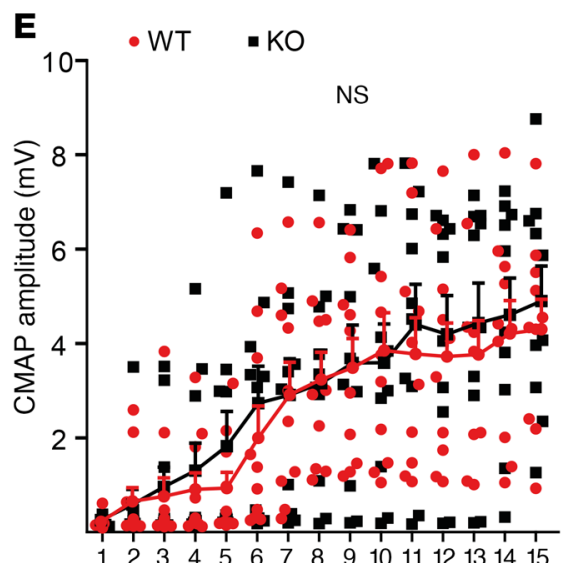

Stimulus intensity (V)
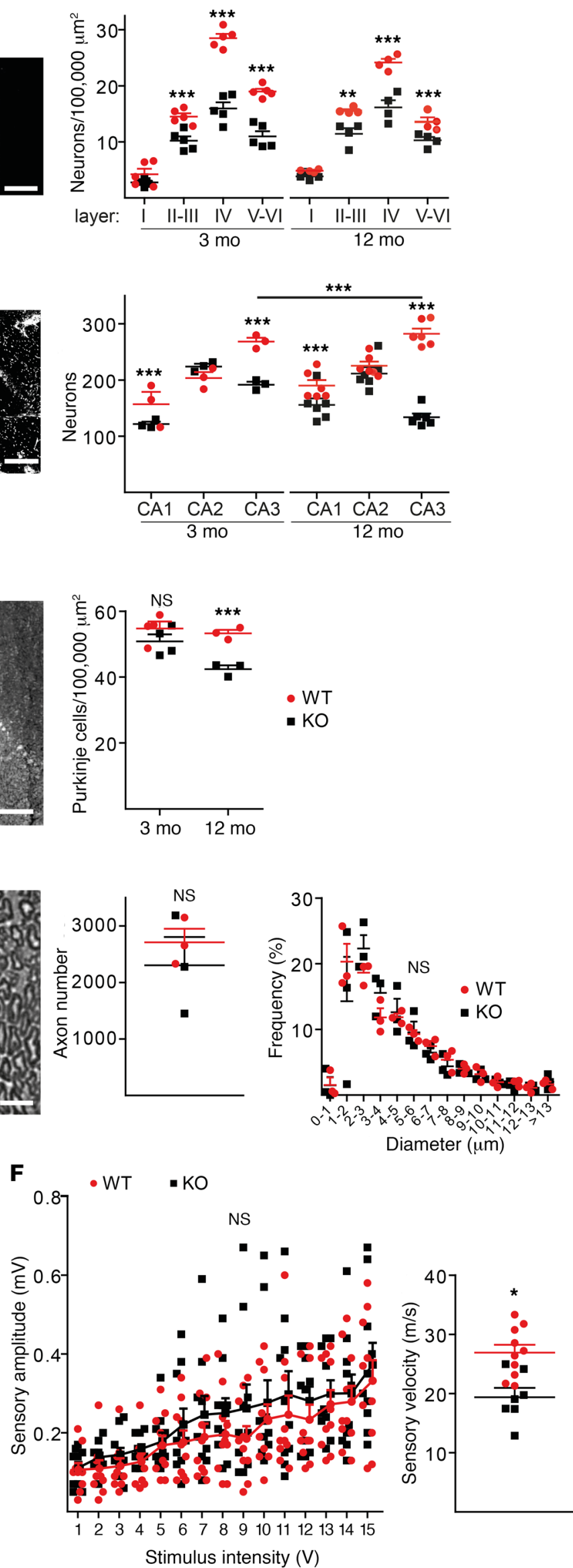
Figure 2. Altered brain development and progressive neuron loss in Gmppa-KO mice. (A) Cortical layering is altered in Gmppa-KO mice. Sagittal sections of the somatosensory cortex of 3-and 12-month-old WT and KO mice were stained for the neuronal marker NeuN and neurons counted layer-wise ( $n=4-6$ mice per group; 2-way ANOVA with Bonferroni post hoc test). Scale bars: $50 \mu \mathrm{m}$. (B) Progressive loss of pyramidal neurons in the hippocampus of Gmppa-KO mice. Hippocampal sections of 3- and 12-month-old WT and KO mice were stained for NeuN and neurons counted in the CA1, CA2, and CA3 region of the hippocampus ( $n=3-6$ mice per group; 2-way ANOVA with Bonferroni post hoc test). Scale bars: $125 \mu \mathrm{m}$. (C) Progressive loss of Purkinje cells in Cmppa-KO mice. Cerebellar sections from 3- and 12-month-old WT and KO mice were stained for calbindin and Purkinje cells counted ( $n=3-4$ mice per group; 2-way ANOVA with Bonferroni post hoc test). Scale bars: $75 \mu \mathrm{m}$. (D) No obvious morphological changes of sciatic nerves of 12-month-old Gmppa-KO mice. Toluidine-blue stained semi-thin cross-sections of sciatic nerves of 12-month-old WT and KO mice. Total axon numbers and the distribution of axons of different diameters were analyzed ( $n=3$ mice per group; unpaired 2-tailed Student's $t$ test for axon number and 1-way ANOVA with Bonferroni post hoc test for distribution). Scale bars: $5 \mu \mathrm{m}$. (E) Amplitudes of distal compound muscle action potentials upon stimulation at the tail root and motor nerve conduction velocities were not changed in 12 -month-old KO mice ( $n=10$ per group; 1 -way ANOVA with Bonferroni post hoc test). (F) Sensory amplitudes upon stimulation at the tip of the tail were not changed in 12-month-old $\mathrm{KO}$ mice ( $n=10$ per group; 1 -way ANOVA with Bonferroni post hoc test); sensory nerve conduction velocities were slightly decreased. Quantitative data are presented as mean \pm SEM with individual data points. ${ }^{*} P<0.05 ;{ }^{* *} P<0.005$; ${ }^{* *} P<0.0005$.

Given that the ECM serves as the interface between myofibers and the external environment and plays an active role in developmental and regenerative processes, we analyzed the localization of typical ECM proteins such as laminin, nidogen, and collagen IV. Laminin showed a more fragmented localization in skeletal muscles from KO mice at 12 months of age (Figure $3 \mathrm{~F}$ and Supplemental Figure 2C), which was not yet evident at 3 months (data not shown). Similar findings were also observed for nidogen (Figure $3 \mathrm{G}$ and Supplemental Figure 2D) and collagen IV (Figure $3 \mathrm{H}$ and Supplemental Figure 2E). Control staining of WT and KO skeletal muscle sections with the respective secondary antibodies omitting primary antibodies did not show significant background staining (Supplemental Figure 2F). The sarcolemma was still intact in 12-month-old KO mice as judged by IgG staining (Supplemental Figure 2F). Electron micrographs, however, indicated that the connection between myofiber basal lamina and endomysial collagen was less compact and tight in KO samples (Figure 3I). Overall protein abundances of laminin, nidogen, and collagen IV were not altered as measured by immunoblot analyses of skeletal muscle lysates (Supplemental Figure 2H).

Mass spectrometry of skeletal muscle lysates identified proteins related to muscle contraction such as the Z-disc-related protein CAPZB as significantly decreased in KO samples (Figure 3J, Supplemental Figure 2I, and Supplemental Table 1. Because of these data, we performed immunofluorescence staining for $\alpha$-actinin, a member of the spectrin family, which anchors myofibrillar actin filaments to Z-discs. Although it is quite unspecific for myopathic disorders, staining for $\alpha$-actinin was less intense and lacked the regular pattern in KO mice at 12 months of age (Figure 3K). Patches with myofibrillar disintegration and Z-band streaming in $\mathrm{KO}$ samples were also evident by electron micros- copy (Figure 3L). In summary, Gmppa-KO mice developed a progressive muscle disease.

GMPPA is an allosteric feedback inhibitor of GMPPB. We have reported that GDP-mannose levels were elevated in lymphoblastoid cells of patients with AAMR (7). A significant increase in GDP-mannose levels was also evident in skeletal muscle of Gmppa-KO mice (Figure 4A). This, in combination with the fact that GMPPA is enzymatically inactive but can bind GDPmannose (16), led us to the hypothesis that GMPPA may act as an allosteric feedback inhibitor of GMPPB and accordingly should interact with GMPPB. To further evaluate this assumption, HEK293T cells were transiently transfected with Myc-tagged GMPPA and FLAG-tagged GMPPB, and subsequently protein complexes were immunoprecipitated from cell lysates. Coprecipitation of GMPPA with GMPPB and vice versa was detectable (Figure 4, B and C). Notably, the disease-associated GMPPA variant p.T334P, located C-terminal of the nucleotidyl transferase domain, did not coprecipitate with GMPPB (Figure 4C). The interaction was preserved for the variant p.G182D, which locates to the nucleotidyl transferase domain. Because GMPPA variants devoid of the C-terminal 205 amino acids did not coprecipitate with GMPPB (Figure 4D), these findings suggest that the interaction requires the intact C-terminal part of GMPPA. Purified recombinant GST-GMPPB pulled down MBP-GMPPA only in the presence of the C-terminal part of GMPPA (Figure 4, E and F, and Supplemental Figure 3A), which points to a direct interaction between both proteins. Pulldown assays further suggest that the binding of the p.G182D and even more of the p.T334P variant of GMPPA to GMPPB was reduced.

The interaction between both WT proteins could be further substantiated by proximity ligation assays (PLAs) on murine skeletal muscle sections with antibodies directed against GMPPA and GMPPB. The PLA signal was detected only in cross-sections of WT skeletal muscle, which indicates proximity of the targets of less than $40 \mathrm{~nm}$ (Figure 4G and Supplemental Figure 3, B and C).

We also measured the GDP-mannose-pyrophosphorylase activity of recombinant GMPPA and GMPPB by colorimetric readout of generated phosphate in the presence of mannose-1-phosphate, GDP-mannose, GTP, and pyrophosphatase (17). GMPPA alone was enzymatically inactive, but inhibited the activity of GMPPB (Figure 4H and Supplemental Figure 3, D-G). This inhibition was preserved for the variant lacking the $\mathrm{N}$-terminal part of GMPPA and absent for the variant lacking its C-terminal part (Figure $4 \mathrm{H}$ ). Moreover, the inhibition of GMPPB by GMPPA depended on GDP-mannose concentration (Figure 4I). Notably, recombinant GMPPA N182D or T334P had no effect on the activity of GMPPB (Figure 4, J and K). Taken together, our data suggest that GMPPA acts as an allosteric feedback inhibitor of GMPPB.

Alpha-DG is hyperglycosylated in skeletal muscle of Gmppa-KO mice. The increase in GDP-mannose levels might cause imbalances in glycosylation reactions in Gmppa-KO mice. We used mass spectrometry to identify alterations in the carbohydrate chains of either $\mathrm{N}$-glycans or $\mathrm{O}$-glycans of skeletal muscle. We found a significant increase in high-mannose $N$-glycans (Figure $5 \mathrm{~A}$ ) and in selected $O$-glycans (Figure $5 \mathrm{~B}$ ) in $\mathrm{KO}$ mice compared with control mice. Therefore, we separated skeletal muscle proteins from 12-month-old mice by SDS-PAGE and probed the blotted proteins 
A

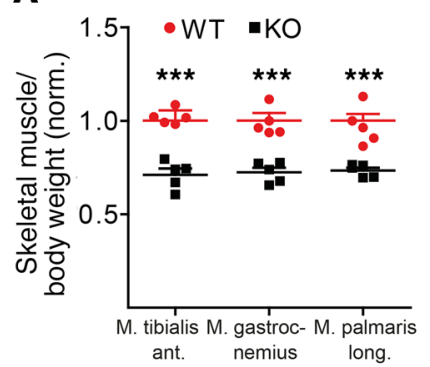

B

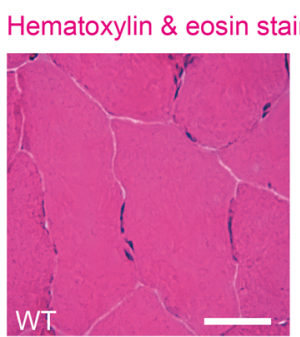

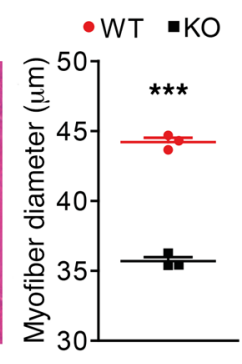
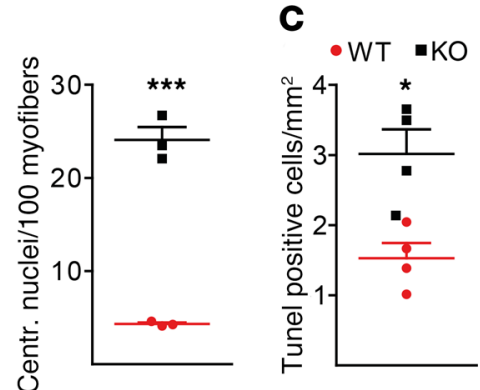

D

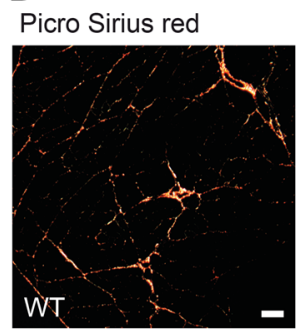

E

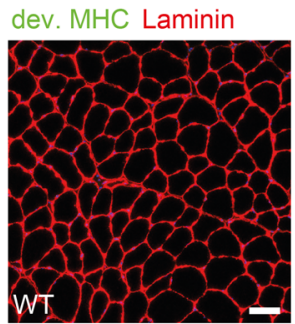

H

Collagen IV

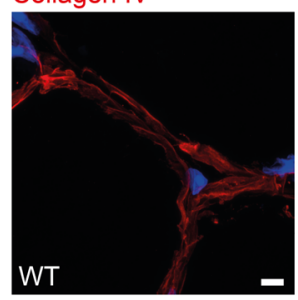

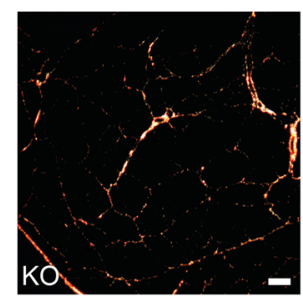

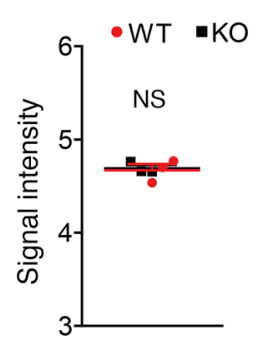

$\mathbf{F}$

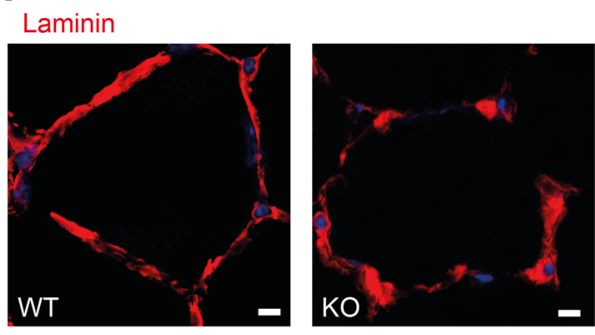

G

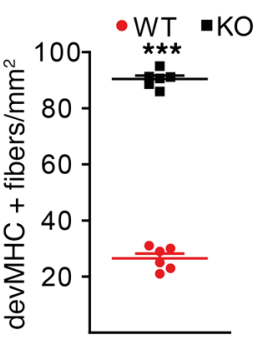

Nidogen
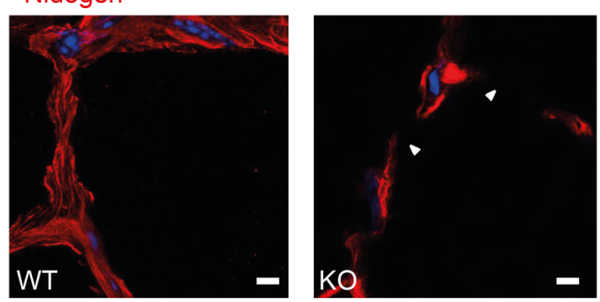

I
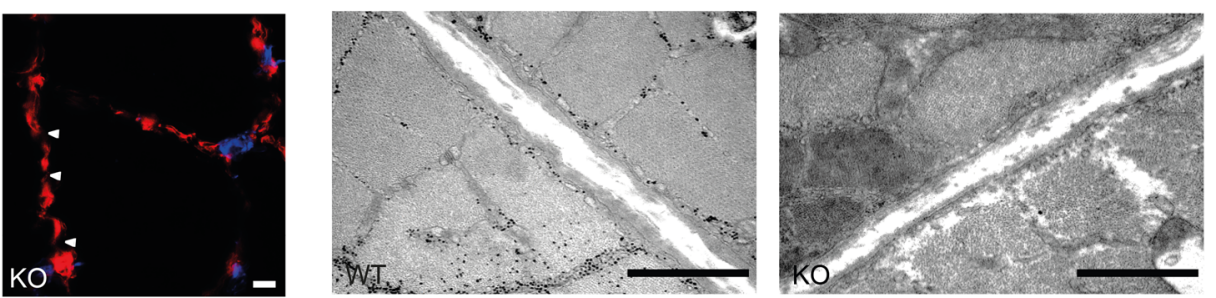

J

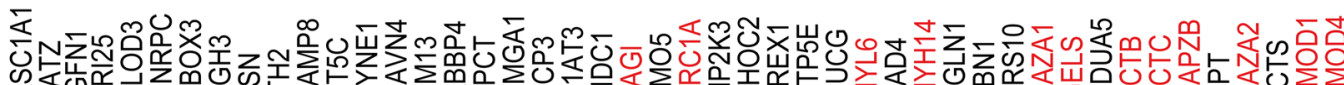

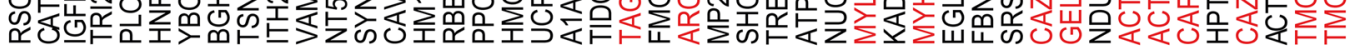

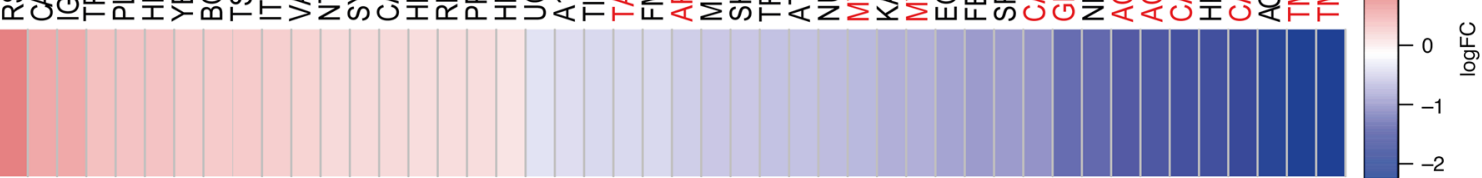

$\mathbf{K}$

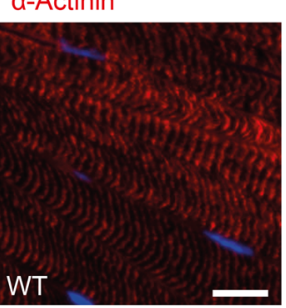

$\mathbf{L}$

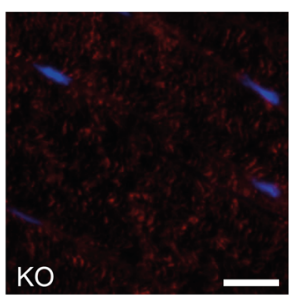

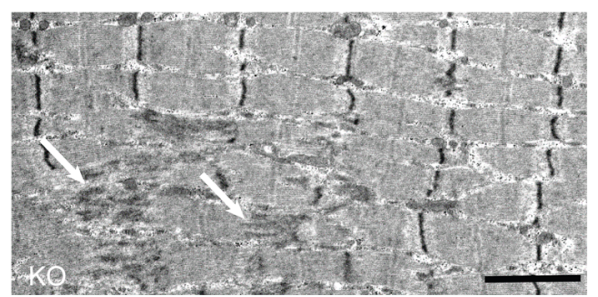


Figure 3. Gmppa-KO mice develop an age-dependent myopathy. (A) Reduced muscle mass in 12-month-old KO mice ( $n=5$ males each; Student's $t$ test). (B) Decreased myofiber diameter and centralized nuclei in skeletal muscle sections of 12-month-old KO mice ( $n=3$ mice per group; unpaired 2-tailed Student's $t$ test). Scale bars: $50 \mu \mathrm{m}$. (C) Increased number of TUNEL-positive nuclei in KO mice ( $n=5$ mice per group; unpaired 2-tailed Student's $t$ test). (D) No relevant skeletal muscle fibrosis at 12 months of age as judged from Picro-Sirius red staining ( $n=3$ mice per group; unpaired 2-tailed Student's $t$ test). Scale bars: $200 \mu \mathrm{m}$. (E) Increased number of developmental myosin heavy chain (green) positive satellite cells in skeletal muscle of 12-month-old KO mice ( $n=6$ mice per group; unpaired 2-tailed Student's $t$ test). Scale bars: $50 \mu \mathrm{m}$. (F-H) Altered distribution of the ECM proteins laminin (F), nidogen (G), and collagen IV (H) in skeletal muscles of 12 -month-old $\mathrm{KO}$ mice. Overviews and control staining with secondary antibodies alone are shown in Supplemental Figure 2. Scale bars: $5 \mu \mathrm{m}$. (I) Less compact and less tight connection of endomysial collagen to myofiber basal lamina in KO samples. Scale bars: $1 \mu \mathrm{m}$. (J) Mass spectrometry analysis of skeletal muscle proteins of 12-month-old WT and KO mice. Proteins significantly upregulated in $\mathrm{KO}$ are shown in red; those downregulated in blue (threshold $q<0.2 ; n=5$ mice per genotype). The red font refers to genes related to muscle contraction. (K) Irregular $\alpha$-actinin pattern in skeletal muscle of 12-month-old KO mice (arrowheads). Scale bars: $10 \mu \mathrm{m} ; n=3$ mice per group. (L) Myofibrillar disintegration with Z-band streaming (arrows). Sarcomeres surrounding the lesion show a normal Z-band pattern. Scale bar: $2 \mu \mathrm{m}$. Quantitative data are presented as mean \pm SEM with individual data points. ${ }^{*} P<0.05 ;{ }^{* *} P<0.0005$.

with lectins, such as concanavalin A (Con A) and peanut agglutinin (PNA) (Figure 5C), which bind to particular sugar sequences of glycoproteins. No obvious difference between genotypes was observed for Con A, which detects $\alpha$-linked mannose, glucose, or $\mathrm{N}$-acetylgalactosamine. In contrast, signals for PNA, which detects nonsialylated $\beta(1-3)$-linked galactose on $N$-acetylgalactosamine residues, were significantly increased in KO samples. The membranes were also probed with antibodies directed against oligomannose and paucimannose. Both were significantly increased in KO lysates as well (Figure 5C). Because of our glycome analysis and the fact that $\alpha-D G$ is heavily glycosylated and its correct glycosylation is a prerequisite for muscle integrity $(18,19)$, we also assessed $\alpha$-DG glycosylation. Indeed, we found that signal intensities were increased in lysates from KO mice when we probed with an antibody directed against a glycosylation-specific epitope of $\alpha$-DG, i.e., IIH6C4 (Figure 5C). Additionally, we observed a shift toward higher molecular weights in KO samples.

We next performed immunostaining of skeletal muscle cross-sections of 12-month-old mice with antibodies directed against oligomannose (Figure 5D and Supplemental Figure 4A), Con A (Figure 5E and Supplemental Figure 4B), PNA (Figure 5F and Supplemental Figure 4C), and the glycosylation-specific epitope of $\alpha$-DG (Figure 5G and Supplemental Figure 4D). In accordance with our immunoblot results, Con A signal intensities did not differ between genotypes, whereas we observed increased staining for oligomannose, PNA, and the glycosylation-specific $\alpha$-DG epitope in KO animals. Signals were absent after deglycosylation of skeletal muscle sections of WT and KO mice with PNGase F (Supplemental Figure 4, E-H). In summary, GDP-mannose levels were increased in skeletal muscle of Gmppa-KO mice, which resulted in the hyperglycosylation of skeletal muscle proteins, including $\alpha$-DG.

Increased $\alpha-D G$ turnover and myotube degeneration upon GMPPA knockdown in myoblasts. To assess whether the myopathy upon dis- ruption of GMPPA is muscle intrinsic, we performed knockdown experiments in primary murine myoblasts followed by differentiation into myotubes. As in Gmppa-KO mice, the knockdown resulted in the hyperglycosylation of $\alpha-\mathrm{DG}$ (Figure 6A).

Myoblasts further allowed us to assess the turnover of $\alpha-D G$ in the presence or absence of GMPPA. We assessed the abundance of $\alpha$-DG with antibodies directed against the $\alpha$-DG core 24 hours after blocking of protein translation with cycloheximide (CHX). Ubiquitin levels confirmed that $\mathrm{CHX}$ treatment was effective. After transfection with scrambled siRNAs, the abundance of $\alpha$-DG decreased by $24 \%$ without and by $47 \%$ upon CHX treatment and knockdown of GMPPA (Figure 6A). This indicated that $\alpha$-DG turnover was increased upon disruption of GMPPA.

To rule out that loss of GMPPA affects myogenesis per se, we addressed whether knockdown of GMPPA in myoblasts interferes with myogenic differentiation in vitro. Primary myoblasts were differentiated into early myotubes (marked by the expression of MyHC and myogenin) and then transfected with siRNAs directed to GMPPA, DAG1, or a scrambled control (Figure 6B). No differences in the fusion index of myoblasts as a marker for myogenic differentiation were observed between the different conditions.

To rule out that early myogenesis is affected by knockdown of GMPPA (20), we also transfected C2C12 myoblasts with siRNA directed to GMPPA, DAG1, or a scrambled control at induction of differentiation (Figure 6C). Again, no differences in the fusion index or myotube diameter (mean \pm SEM: siDAG1 13.76 $\pm 0.45 \mu \mathrm{m}$, siScr $12.22 \pm 0.48 \mu \mathrm{m}$, siGMPPA $11.19 \pm 0.27 \mu \mathrm{m} ; P>0.05)$ were observed when GMPPA was knocked down.

Because Gmppa-KO mice develop a progressive myopathy, we asked whether myotubes are affected by GMPPA knockdown. C2C12 cells were differentiated into late myotubes and then transfected with siRNA against GMPPA, DAG1, or a scrambled control (Figure 6D). Knockdown of GMPPA caused a decrease in myotube diameter compared with the control, suggesting that loss of GMPPA resulted in the atrophy of myotubes/myofibers. As expected, we also observed a decrease in myotube diameter after knockdown of DAG1. Taken together, knockdown of GMPPA in myoblasts increased its turnover and affected the size of late myotubes.

Hyperglycosylation and decreased abundance of $\alpha-D G$ in patients with $A A M R$. We next addressed whether our in vitro findings also apply in vivo. Indeed, the signals with the antibody directed against the $\alpha-D G$ core epitope were reduced in skeletal muscle sections of Gmppa-KO mice, whereas $\beta$-DG abundance was unchanged (Figure 7, A and B, and overviews shown in Supplemental Figure 5A). Because signal intensities for the $\alpha$-DG core were reduced in $\mathrm{KO}$ samples after prior deglycosylation, we conclude that the core epitope was not masked by hyperglycosylation (Supplemental Figure 5B). Decreased abundance of the $\alpha$-DG core was further confirmed by immunoblot analyses of both native (Figure 7C) and deglycosylated (Supplemental Figure 5C) skeletal muscle protein lysates.

We also had access to muscle biopsies from 2 adult sisters with AAMR syndrome homozygous for the GMPPA variant p.R373P (21). We found an increased staining for the glycosylation-specific epitopes of $\alpha$-DG (IIH6C4, VIA4) in patient samples compared with a healthy control (Figure 7, D and E), and the signal intensities for the $\alpha$-DG core protein were reduced (Figure 
A

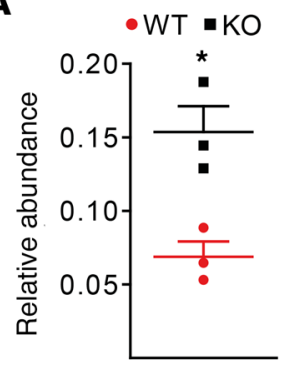

B

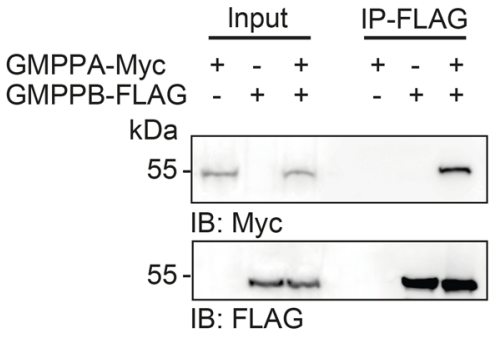

C

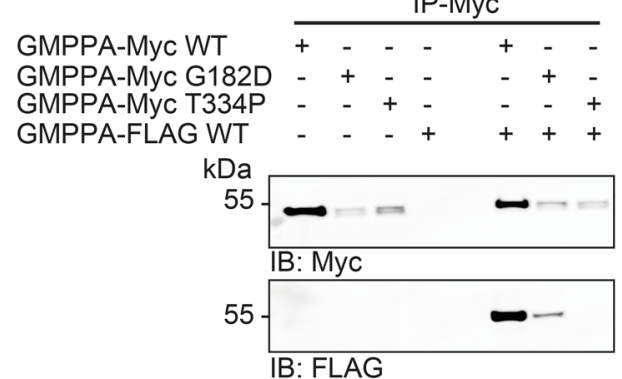

D

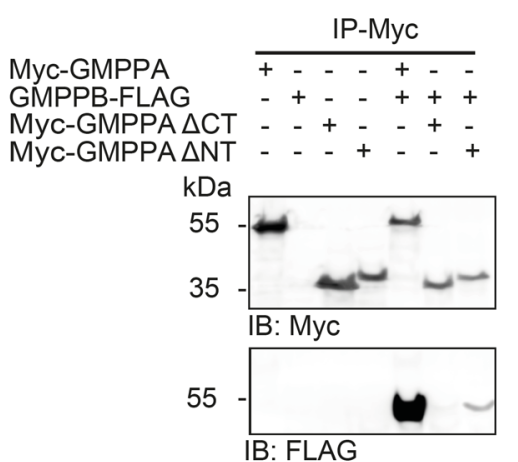

\section{E}

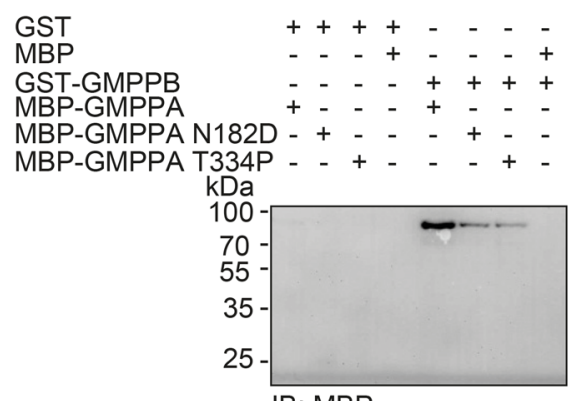

IB: MBP

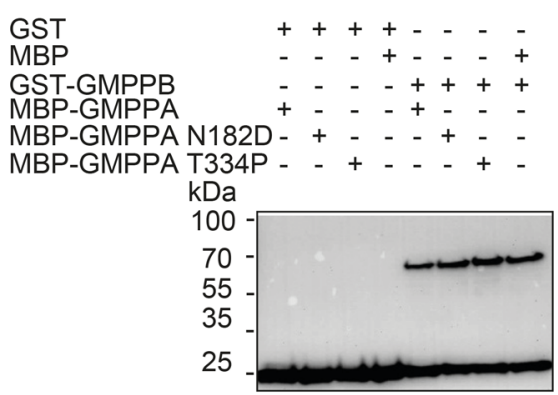

IB: GST
$\mathbf{F}$

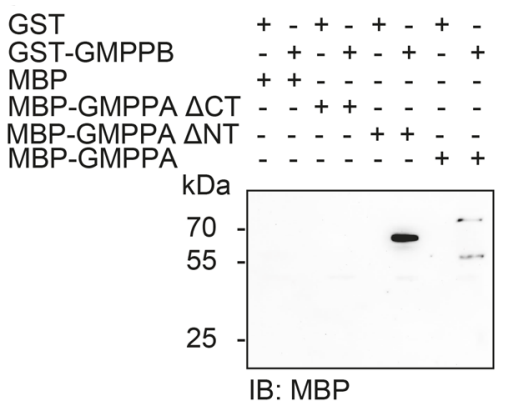

G

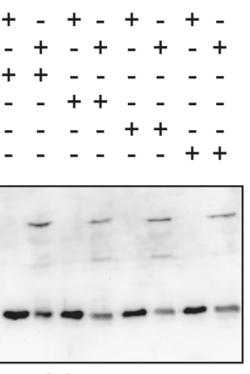

IB: GST
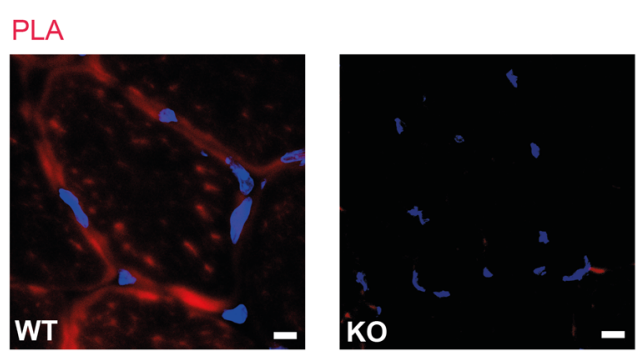

H

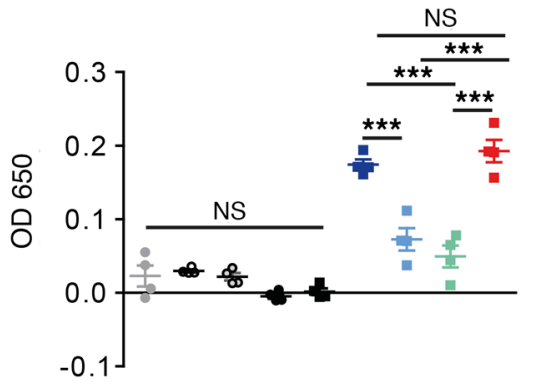

- GST-GMPPA

- GST-GMPPA $\triangle N$ - GST-GMPPA $\triangle C$ - GST

MBP

- MBP-GMPPB

MBP-GMPPB + GST-GMPPA - MBP-GMPPB + - MBP-GMPPB +

J

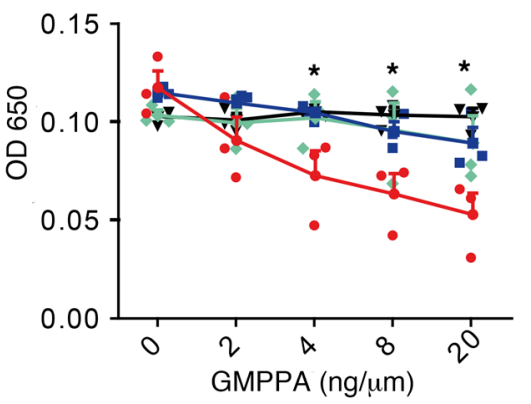

- GST-GMPPB + MBP-GMPPA

- GST-GMPPB + MBP-GMPPA N182D

- GST-GMPPB +

MBP-GMPPA T334P -GST-GMPPB + MBP

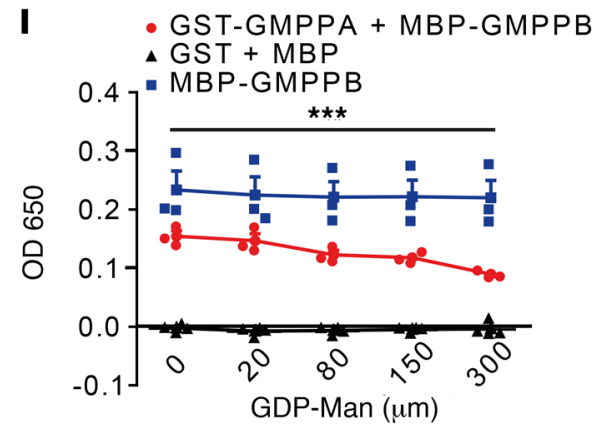

K

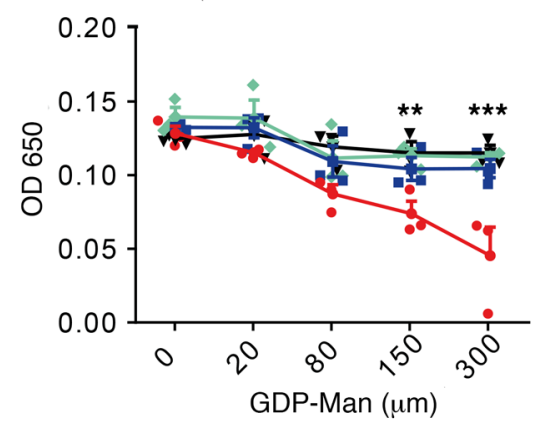


Figure 4. GMPPA is an allosteric inhibitor of GMPPB. (A) GDP-mannose was increased in skeletal muscles of 8-month-old KO mice ( $n=3$ mice per group; unpaired 2-tailed Student's $t$ test). (B) Overexpressed GMPPA-Myc and GMPPB-FLAG coprecipitated. (C) Upon heterologous expression with GMPPB, the N-terminal GMPPA variant G182D was detected but not the C-terminal variant T334P. (D) GMPPA devoid of the C-terminal aa 206-420 $(\triangle C T)$ did not interact with GMPPB; GMPPA devoid of the N-terminal aa 1-205 ( $\triangle N T$ ) still coprecipitated with GMPPB. (E) Pulldown of recombinant GST-GMPPA WT, -GMPPA N182D, -GMPPA T334P, and MBP-GMPPB. (F) Pulldown of recombinant MBP-CMPPA deletion constructs and GST-GMPPB. (C) Red signals indicate proximity of GMPPA and GMPPB of $40 \mathrm{~nm}$ or less in WT but not in KO skeletal muscle in proximity ligation assays (PLAs). Scale bars: $5 \mu \mathrm{m}$. Additional controls are shown in Supplemental Figure 3. (H) Recombinant purified MBP-GMPPB showed enzymatic activity, which was inhibited by an equal amount of recombinant GST-GMPPA in the presence of $80 \mu \mathrm{M}$ GDP-mannose ( $n=3$ experiments; 1-way ANOVA with Bonferroni post hoc test). (I) Inhibition of GMPPB activity by GMPPA WT increased with higher GDP-mannose concentrations ( $n=3$ experiments; 2-way ANOVA with Bonferroni post hoc test). Controls are shown in Supplemental Figure 3. (J) The enzymatic activity of recombinant GMPPB $(20 \mathrm{ng} / \mu \mathrm{L})$ was inhibited by increasing amounts of recombinant GMPPA WT but not by disease-associated variants N182D and T334P ( $n=3$ experiments; 2-way ANOVA with Bonferroni post hoc test). (K) The enzymatic activity of recombinant GMPPB was inhibited in a GDP-mannose-dependent manner in the presence of recombinant GMPPA WT, but not in the presence of GMPPA N182D and T334P ( $n=3$ experiments; 2-way ANOVA with Bonferroni post hoc test). Quantitative data are presented as mean \pm SEM with individual data points. ${ }^{*} P<0.05$; ${ }^{* *} P<0.005$; ${ }^{* *} P<0.0005$.

7F). In agreement with our findings obtained in Gmppa-KO mice, $\beta$-DG signals did not differ (Figure $7 G$ ), and the distribution of laminin was less homogenous compared with the control (Figure $7 \mathrm{H}$ ). Immunoblot analysis of skeletal muscle lysates showed a reduction of GMPPA abundance and increased signal intensities for both oligomannose and PNA, whereas signals for Con A and laminin were unchanged (Figure 7I), similar to our findings in Gmppa-KO mice. Moreover, signal intensities with the antibodies IIH6C4 and VIA4, which are directed against glycosylation-specific epitopes of $\alpha$-DG, clearly confirmed $\alpha$-DG hyperglycosylation in patients with AAMR. Signal intensities with the antibody directed against the $\alpha$-DG core were reduced (Figure 7I). As in KO mice, the immunostaining pattern for the Z-disc protein $\alpha$-actinin was less organized in biopsies from patients with AAMR and lacked the regular pattern observed in control samples (Figure 7J). In agreement, the ultrastructural analyses showed disarrayed filaments and loss of the continuity of the Z-discs in patients with AAMR compared with the control (Figure $7 \mathrm{~K}$ ). In short, the relevance of our findings in Gmppa-KO mice was confirmed by the analysis of skeletal muscle biopsies from patients with AAMR syndrome.

Motor impairment of Gmppa-KO mice can be rescued by dietary mannose restriction. Of note, serum mannose concentrations were significantly increased in 3-month-old untreated $\mathrm{KO}$ mice (Figure 8A). Because mannose is easily taken up in the gastrointestinal tract (22), we considered that mannose serum levels may be normalized by dietary mannose restriction. Control and Gmppa-KO mice were either fed with a nominally mannose-free diet, in which mannose was replaced by sucrose, or the regular chow starting from P14 up to 12 months of age. The mannose serum concentration in WT mice was largely unaffected by the diet, whereas mannose levels almost normalized in 3-month-old treated KO mice (Figure 8A).

Contextual learning in the fear-conditioning paradigm (Figure $8 \mathrm{~B}$ ) and the abnormal cortical layering (Figure $8 \mathrm{C}$ and Supplemental Figure 6A) were not improved by dietary intervention at 3 months of age. The progressive loss of hippocampal neurons (Figure 8D and Supplemental Figure 6B) and Purkinje cells (Figure $8 \mathrm{E}$ and Supplemental Figure 6C), however, was largely attenuated by the diet. The deterioration of motor functions was almost completely prevented in Gmppa-KO mice as assessed by the footbase angle (Figure 8F), the latency to fall from a wire mesh (Figure $8 \mathrm{G}$ ), and the weights test (Figure $8 \mathrm{H}$ ). The muscle mass was also almost normalized in treated KO mice (Supplemental Figure 6D). Although centralized nuclei were drastically increased in the untreated $\mathrm{KO}$ cohort, they were almost normalized by dietary intervention (Figure 8I and Supplemental Figure 6, G and H). The altered distribution of laminin signals and the decreased mean myofiber diameter were rescued as well by dietary intervention (Figure 8J and Supplemental Figure 6, G and H).

Signal intensities of Western blots of skeletal muscle lysates probed with PNA and antibodies directed against paucimannose, oligomannose, the $\alpha$-DG core, and its glycosylation-specific epitope revealed that the mannose-free diet almost normalized the levels and glycosylation status of these proteins in Gmppa-KO mice (Figure 8K). Increased phosphorylation of ERK 1/2, indicating that MAPK/ERK signaling was activated in KO muscle, largely normalized as well. These results showed that neurodegeneration and motor impairment could be largely prevented by postweaning dietary mannose restriction of Gmppa-KO mice but did not improve defects of brain development.

\section{Discussion}

Up to now, the function of GMPPA and thus the pathophysiology of AAMR syndrome remained largely unclear, because GMPPA itself lacks enzymatic activity (6). This is explained by a 2 aa insertion in a highly conserved motif near the catalytic pocket of GMPPA's N-terminal nucleotidyl-transferase domain (aa $3-194)(16,23)$, which is shared with other enzymes that transfer nucleotides onto phosphosugars (24). The absence of GDPmannose-pyrophosphorylase activity for GMPPA was also confirmed by our data. Because GMPPA can still bind GDP-mannose (25) and the presence of GMPPA inhibited the activity of GMPPB in a GDP-mannose dependent manner, our findings further suggest that GMPPA acts as an allosteric feedback inhibitor of GMPPB. In agreement with this function, GMPPA and GMPPB directly interact with each other. Notably, this interaction requires the intact C-terminal part that includes a hexapep domain (aa 286319), which is likewise found in several members of the transferase families (26). Interestingly, the interaction was abolished in the disease-associated C-terminal GMPPA variant p.T334P, which lacks inhibition of GMPPB activity.

As an allosteric feedback inhibitor of GMPPB, loss of GMPPA is predicted to result in increased GDP-mannose levels, which was indeed observed in skeletal muscles of Gmppa-KO mice. Because the increase in GDP-mannose levels can cause imbalances in glycosylation reactions by inhibiting enzymes with the use of other nucleoside diphosphate sugar sugars or by favoring reactions displaying a 


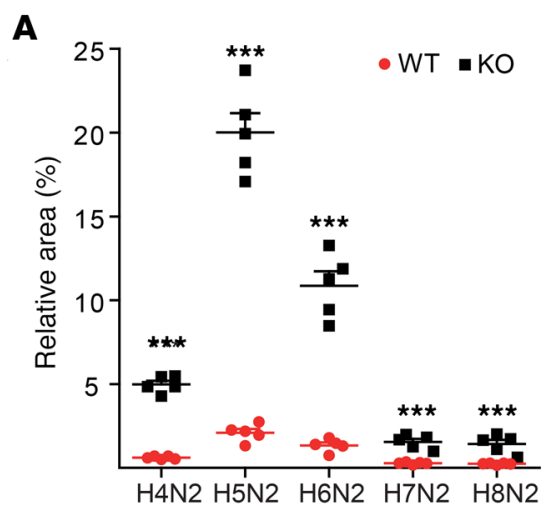

C
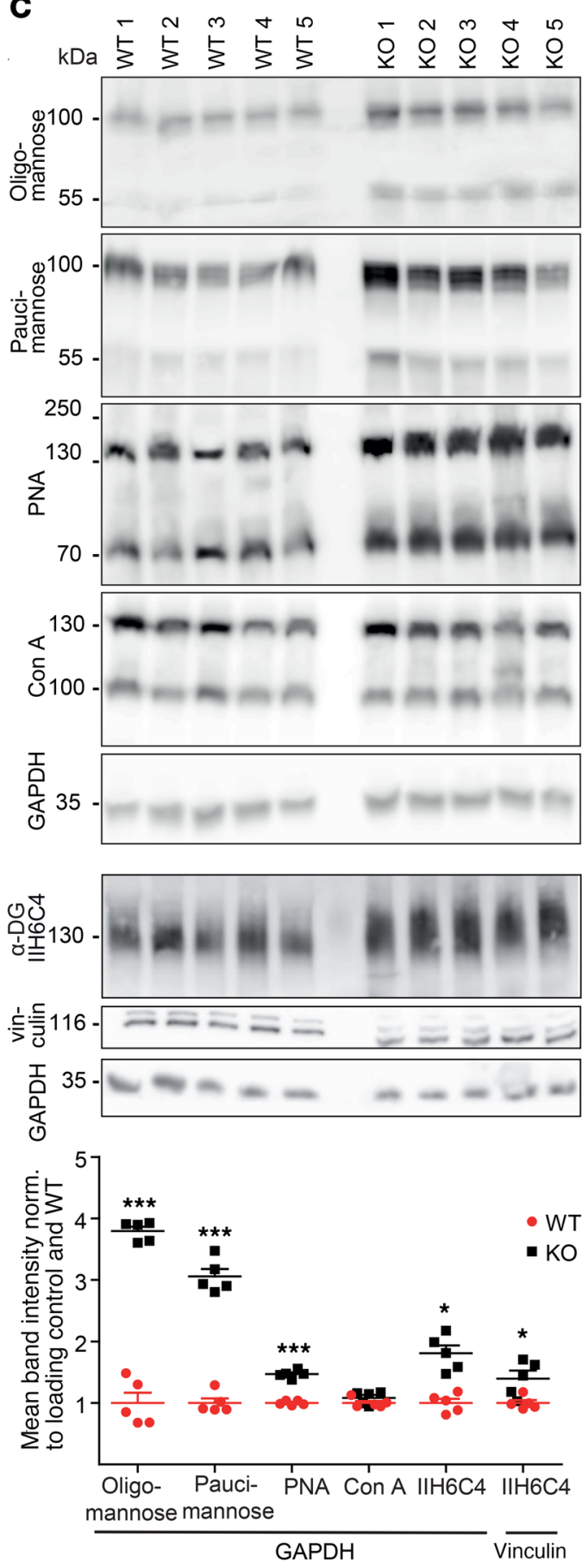

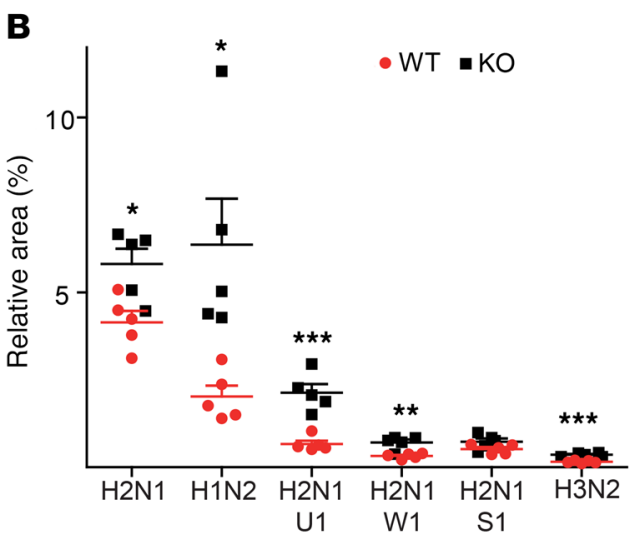

D Oligomannose

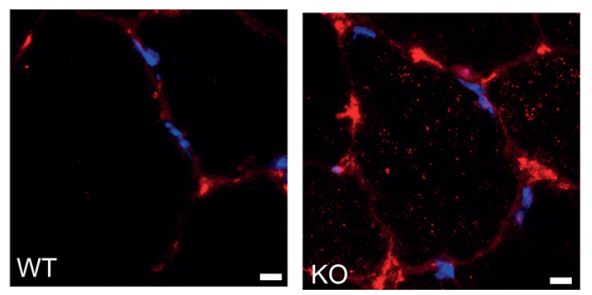

E
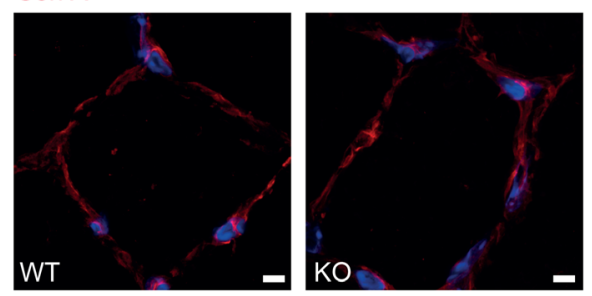

$\mathbf{F}$
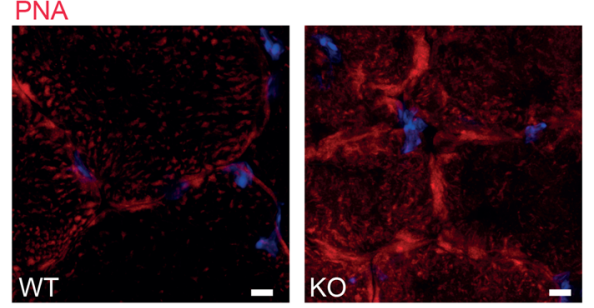

G

$\alpha-D G ~ I H 6 C 4$
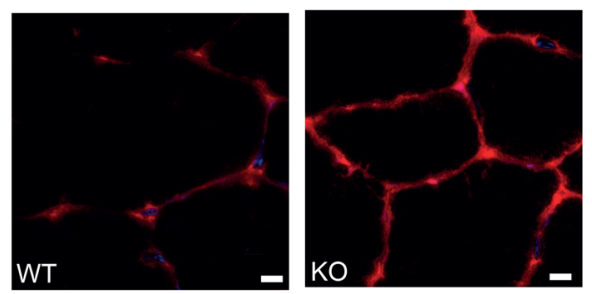
Figure 5. Loss of GMPPA causes $\alpha$-DG hyperglycosylation. (A and B)

Skeletal muscle glycome analysis of 12-month-old fasted mice ( $n=5$ per group; 1-way ANOVA with Bonferroni post hoc test). Relative peak areas from MALDI-TOF mass spectra. (A) $\mathrm{N}$-glycans, (B) $\mathrm{O}$-glycans. $\mathrm{H}$ : hexose, $\mathrm{N}$ : N-acetylhexosamine. S: N-acetylneuraminic acid, U: glucuronic acid, W: glucuronic acid with 1 sulphate group. (C) Immunoblot analyses of skeletal muscle lysates showed increased signals for oligomannose, paucimannose, peanut agglutinin (PNA), and the glycosylation-specific $\alpha$-DG epitope IIH6C4 in samples from 12-month-old KO mice. Signals for concanavalin A (Con A) were unchanged. Vinculin and GAPDH served as loading controls ( $n=5$ mice per group; 2-way ANOVA with Bonferroni post hoc test). (D-G) Immunostaining of skeletal muscle sections from 12-month-old WT and KO mice ( $n=3$ per group). Scale bars: $5 \mu \mathrm{m}$. Overviews and control staining after PNGase $F$ treatment are displayed in Supplemental Figure 4. Additional loading controls are displayed in Supplemental Figure 7. (D) Increased oligomannose signals in KO samples. (E) Con A signals were unchanged. (F) PNA signals were increased in KO samples. (C) Signals for IIH6C4 were increased in KO samples. Quantitative data are presented as mean $\pm \mathrm{SEM}$ with individual data points. ${ }^{*} P<$ $0.05 ;{ }^{*} P<0.005$; ${ }^{* *} P<0.0005$.

high Michaelis-Menten constant for this NDP sugar, we performed a glycome analysis by mass spectrometry and found a significant increase in high-mannose $\mathrm{N}$ - and $\mathrm{O}$-glycan structures. Also, the signals for the carbohydrate-binding lectin PNA as well as for antibodies directed against oligo- and paucimannose were increased in skeletal muscle protein lysates or tissue sections of Gmppa-KO mice. More specifically, we identified $\alpha-D G$ as a hyperglycosylated protein in skeletal muscles of Gmppa-KO mice, which we also confirmed in skeletal muscle biopsies from patients with AAMR.

Typically, dystroglycanopathies either result from primary dystroglycan defects or from $\alpha$-DG hypoglycosylation, which weakens $\alpha$-DG's binding to the ECM and thus causes membrane fragility (27). The binding depends on the repeating disaccharide (-3-xylose-alpha1,3-glucuronic acid-beta1-), which is attached by like-acetylglucosaminyl transferase (LARGE) $(28,29)$. Notably, LARGE is mutated in some forms of muscular dystrophies (30). Its transgenic overexpression resulted in $\alpha$-DG hyperglycosylation and thereby increased laminin binding (31). Therefore, it was proposed that LARGE overexpression might be a therapeutic strategy for dystroglycanopathies (31). However, LARGE overexpression decreased maximal muscle force (31) and worsened the muscle pathology in fukutin-related protein knockdown mice (32). In light of these findings, this strategy might be dangerous because the hyperglycosylation of $\alpha$-DG may compromise the maintenance of skeletal muscle fibers.

We considered that the turnover of hyperglycosylated $\alpha-D G$ may be increased and may thus lead to less abundance of the $\alpha-D G$ core protein. Indeed, less abundance of the $\alpha-D G$ core protein was suggested by our analyses of skeletal muscles of $\mathrm{KO}$ mice and skeletal muscle biopsies from patients with AAMR. This suggests that there is even more glycosylation per core protein, as suggested by our Western blots. Excluding the possibility that the hyperglycosylation may mask the $\alpha$-DG core epitope, the $\alpha$-DG core abundance was decreased in KO mice after enzymatic deglycosylation of skeletal muscle samples. Moreover, siRNA-mediated knockdown of GMPPA in myoblasts caused $\alpha$-DG hyperglycosylation and increased its turnover, as judged by immunoblot analysis after blocking protein translation. The reduced size of myotubes upon knockdown of GMPPA is in agreement with muscle-intrinsic effect of GMPPA loss of function. The decreased abundance and the hyperglycosylation of $\alpha$-DG may explain the irregular distribution of its ECM binding partners such as laminin.

The balanced supply of GDP-mannose as a sugar donor for both $\mathrm{N}$ - and $\mathrm{O}$-glycosylation (6) requires the interplay between GMPPA and GMPPB, which catalyzes the production of GDP-mannose from mannose-1-phosphate and GTP. Mutations in phosphomannomutase 2 (PMM2) (33), which converts mannose-6-phosphate to mannose-1-phosphate, and mutations in phosphomannose isomerase (PMI) (34), which facilitates the interconversion of fructose 6-phosphate and mannose-6-phosphate, decrease the availability of GDP-mannose and thus result in the hypoglycosylation of glycoproteins. Mannose is easily taken up in the gastrointestinal tract (35) and together with glucose-derived mannose as well as mannose released from glycans undergoing degradation (36) contributes to the mannose pool used for glycoconjugate synthesis (35). Thus, external mannose supplementation rescued the complex symptoms of patients (34) with PMI defects and was successful in treating PMM2 hypomorphic mice (37). In Gmppa$\mathrm{KO}$ mice, however, we found serum mannose concentrations to be strongly increased, which may reflect increased release from glycans and/or increased generation of mannose from glucose. Although only approximately $2 \%$ of mannose entering the cell is used for glycosylation (35), the higher systemic mannose levels may contribute to the larger pool of GDP-mannose in Gmppa-KO mice and thus hyperglycosylation. Therefore, we assessed whether increased systemic mannose concentration and the hyperglycosylation in Gmppa-KO mice can be mitigated by dietary mannose restriction and fed control and $\mathrm{KO}$ mice a diet in which mannose was replaced by sucrose starting at P14. This almost normalized the mannose concentration in the serum of 3-month-old $\mathrm{KO}$ mice. Moreover, $\alpha$-DG hyperglycosylation and the decrease in the abundance of the $\alpha$-DG core largely normalized. Importantly, the deterioration of motor performance and neuron loss were also largely prevented by dietary intervention. Given that cortical development is already largely established at P14 in mice (38), when we started with dietary mannose restriction, it came as no surprise that cortical layering and cognitive functions as assessed by fear conditioning were not improved. Therefore, it will be important to address whether dietary intervention in pregnant mice can be beneficial for cortex development and cognitive functions of $\mathrm{KO}$ offspring in future studies.

Taken together, we here established a role of GMPPA as an allosteric feedback inhibitor of GMPPB. This resulted in increased GDP-mannose tissue levels in GMPPA deficiency and increased the incorporation of mannose into glycoproteins, including $\alpha$-DG. Because the hyperglycosylation increased the turnover of $\alpha-D G$, it lowered its overall abundance, which likely contributed to GMPPA-associated myopathy. Dietary mannose restriction was successful in correcting $\alpha-D G$ glycosylation and abundance and largely prevented neuron loss and deterioration of motor functions in Gmppa-KO mice.

\section{Methods}

To study the function of GMPPA and the consequences of its disruption, we investigated Gmppa-KO mice and cell culture models. Exper- 
A siDAG1 siScr siGMPPA
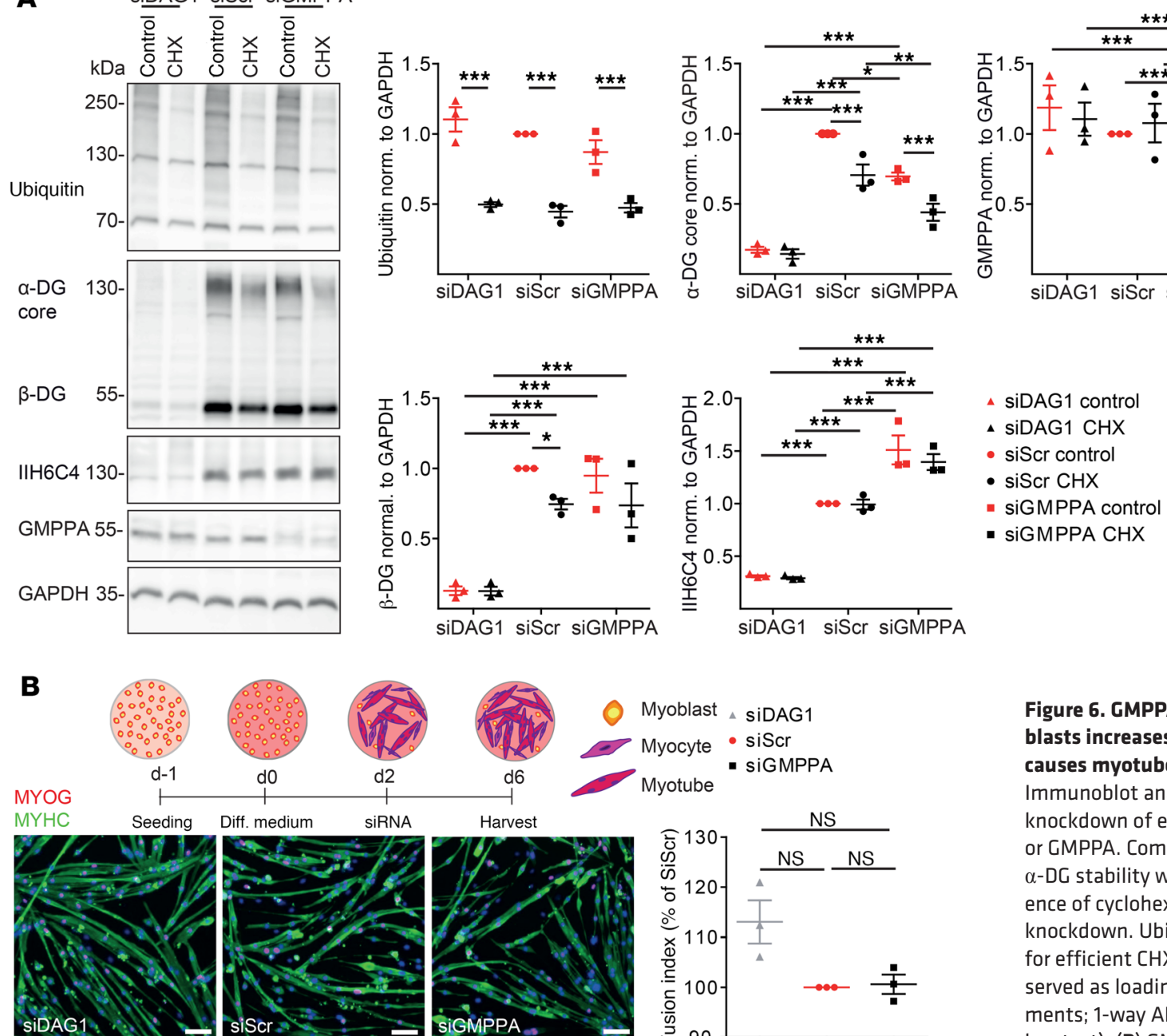

C

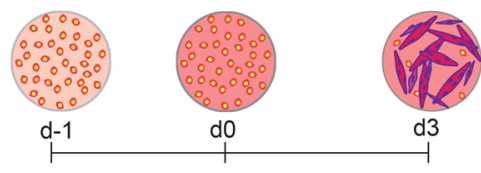

MYOG
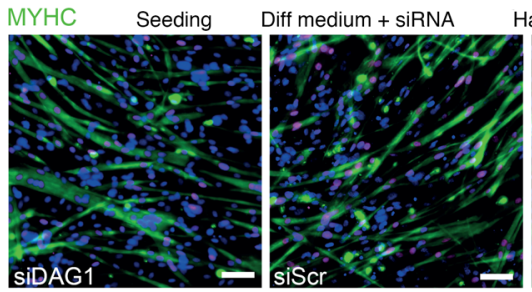

Harvest

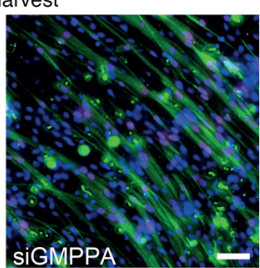

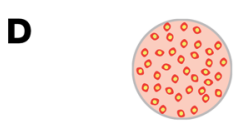

d-1

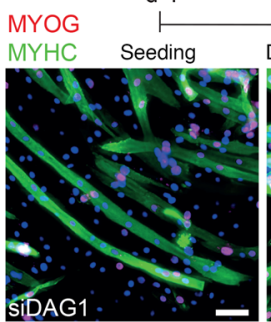

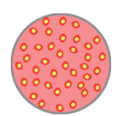

dO

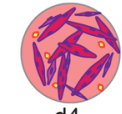

d4

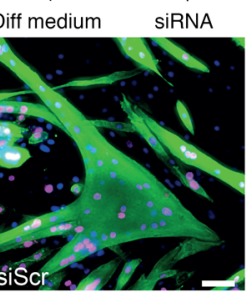

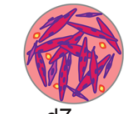
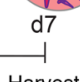

Harvest

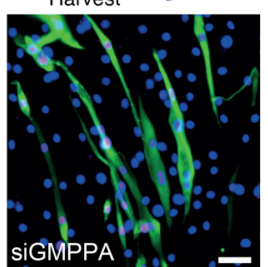

Myoblast ^ siDAG1

Myocyte $\bullet$ siSc Myotube

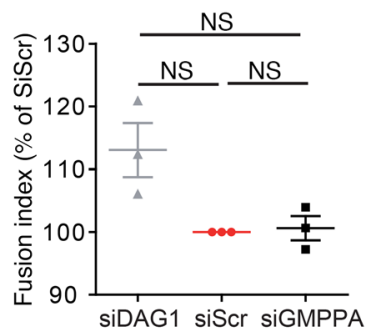

siDAG1 siScr siGMPPA
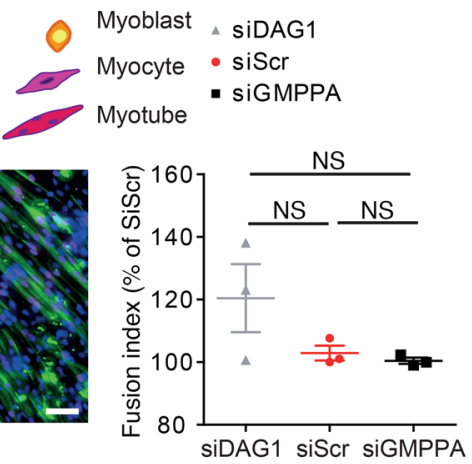

siDAG1 siScr siGMPPA

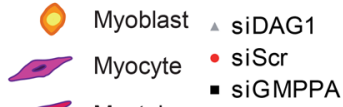

Myotube

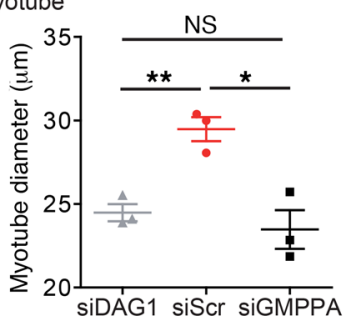

Figure 6. GMPPA knockdown in myoblasts increases $\alpha$-DC turnover and causes myotube degeneration. (A) Immunoblot analyses confirmed efficient knockdown of either dystroglycan (siDag1) or GMPPA. Compared with controls (siScr), $\alpha$-DG stability was decreased in the presence of cycloheximide (CHX) upon GMPPA knockdown. Ubiquitin served as a control for efficient $\mathrm{CHX}$ treatment. GAPDH served as loading control ( $n=3$ experiments; 1-way ANOVA with Bonferroni post hoc test). (B) GMPPA knockdown did not affect the differentiation of primary myoblasts ( $n=3$ experiments; 1 -way ANOVA with Bonferroni post hoc test). Experimental design, representative images of primary mouse myoblasts stained for myogenin (MYOG) or myosin heavy chain (MYHC), and quantification of the fusion index. (C) Knockdown of GMPPA did not affect the differentiation of the myoblast cell line C2C12 ( $n=3$ experiments; 1-way ANOVA with Bonferroni post hoc test). Experimental design, representative images stained for MYOC and MYHC, and quantification of the myotube diameter. (D) The size of C2C12-derived myotubes was altered upon knockdown of GMPPA ( $n=3$ experiments; 1-way ANOVA with Bonferroni post hoc test). Experimental design, representative images of differentiated C2C12 cells, and quantification of myotube diameter. Nuclei are stained in blue. Scale bars (B-D): $70 \mu \mathrm{m}$. Quantitative data are presented as mean \pm SEM with individual data points. ${ }^{*} P<0.05$; ${ }^{* *} P$ $<0.005$; ${ }^{* *} P<0.0005$. 
iments were performed in a C57BL/6 background in the fourth generation. Mice were housed in a 12-hour light/12-hour dark cycle and had access to mouse chow ad libitum (9\% fat, $24 \%$ protein, $67 \%$ carbohydrates including $1 \%$ of free mannose). Treatment cohorts were fed with nominally mannose-free food, in which free mannose had been replaced by saccharose. Littermates of the same sex were randomly assigned to experimental groups. Endpoints were termination of treatment and moribundity for mice in accordance with IACUC guidelines. Experiments were conducted in a blinded manner as to cell and mouse and human genotypes. Figure legends include details of replicate experiments used to generate data sets. All animal experiments were approved by the Thüringer Landesamt für Lebensmittelsicherheit und Verbraucherschutz (TLLV). In behavioral experiments for the initial characterization, groups comprised 6 female and 6 male mice per genotype. For the intervention study, the groups consisted of 6 females and 5 males per condition and genotype. For tissue extraction and histology, male mice were used.

Targeted inactivation of the murine GMPPA gene. To disrupt Gmppa in mice, we obtained the EUCOMM EPD0621_7_G03 embryonic stem cell clone, which harbors a gene-trap cassette following exon 4 and 6 of the Gmppa gene. This clone was injected into C57BL/6 donor blastocysts and transferred into foster mice. The resulting chimeric mice were subsequently mated with FLPe-deleter (12) and Cre-deleter (13) mice in order to generate homozygous KO mice devoid of exon 5. ES cell clones and tail biopsies from chimeric mice were analyzed by Southern blot using EcoRI and a probe of $453 \mathrm{bp}$ binding to Intron 2 of the Gmppa locus.

Motor performance and fear conditioning. Beam-walk test: Mice were trained to walk on a horizontal beam. The foot-base angle was measured at toe-off positions of the hind paws using single video frames from recordings of beam-walking mice with Image (NIH) (14). For statistical analyses, the mean of 2 independent trials was taken.

Kondziela's inverted screen test: The mouse was placed in the center of a wire mesh, the screen was inverted, and the time was taken until the mouse fell off the screen. If the mouse did not fall off the screen, the experiment was terminated after 70 seconds (39).

Weights test: A mouse was held on its tail base and allowed to grip weights $(2.8 ; 9.2 ; 15.4 ; 19 ; 34.2 ; 42.5 ; 50 ; 60.4 ; 73 ; 86.5$ and 103.0 g) with the forepaws. If the mouse was able to hold the weight for 3 seconds or more, the procedure was repeated with the next heavier weight. If the mouse failed to hold the weight for 3 seconds, the procedure was repeated for the same weight. If the mouse failed at 3 consecutive events, the experiment was terminated.

Fear conditioning: Mice were placed in a chamber $(\mathrm{d} 17 \times \mathrm{w} 17 \times$ h $25 \mathrm{~cm}$, Plexiglas wall, 4 lux light, 70\% ethanol, fan speed 100\%) and allowed to explore the surrounding area for 180 seconds. A tone was played for the following 20 seconds ( $9 \mathrm{kHz}$, volume $20 \%, 80 \mathrm{~dB}$ ) paired with a foot shock (unconditioned stimulus, $0.7 \mathrm{~mA}$ for $2 \mathrm{sec}-$ onds) applied in the last 2 seconds via the metal grid. After an additional 60 seconds, mice were returned to their home cages. After 24 hours, the cued test was performed to assess the tone-shock association. Mice were placed in a differently shaped box with altered color pattern (Caro patterned wall, white floor), lighting (2 lux), odor ( $3 \%$ acetic acid), and fan speed (50\%). Mice were allowed to explore the new area for 180 seconds before the tone was applied for $180 \mathrm{sec}-$ onds. After an additional 60 seconds, mice were transferred back into their home cages and allowed to relax for 2 hours. After 2 hours, they were placed in the same context as during acquisition (Plexiglas walls, metal grid floor, lightening 4 lux, odor $70 \%$ ethanol, fan speed $100 \%$ ), and observed for 180 seconds. Animals were video recorded the whole time for automatic detection of freezing by ANY-maze software (Stoelting). For freezing detection, videos were manually analyzed. Freezing time was presented as percentage of the investigated 60-second intervals.

Electrophysiological analysis of peripheral nerves. Anesthetized mice $(100 \mathrm{mg} / \mathrm{kg}$ ketamine and $16 \mathrm{mg} / \mathrm{kg}$ xylazine) were placed on a heating pad. One needle electrode (Science Products, WE30030.1H10) was inserted near the base of the tail and a second one $30 \mathrm{~mm}$ distal to the stimulation site close to the tip of the tail. For the analysis of motor fibers, the stimulus was applied via the proximal electrode and the response recorded with the distal electrode. For the analysis of sensory fibers, the stimulus was applied via the distal electrode and recorded from the proximal electrode. The amplitude of the rectangular stimulation pulses (duration $0.1 \mathrm{~ms}$ ) was increased stepwise from $1 \mathrm{~V}$ to $15 \mathrm{~V}$. Sum action potentials were filtered (high pass $20 \mathrm{~Hz}$, low pass $10 \mathrm{kHz}$ ), digitized (sampling $20 \mathrm{kHz}$ ), and averaged.

Real-time quantitative PCR. RNA was isolated by TRIzol-chloroform extraction. RNA was reverse-transcribed using the SuperScript III Transcriptase kit (Invitrogen). For quantitative PCR (qPCR), the innuMix qPCR MasterMix (Analytik Jena) and TaqMan Gene Expression Assays (Thermo Fisher Scientific) for Gapdh (4331182, assay identifier: Mm99999915_g) and Gmppa (4331182, assay identifier: Mm00505084_m1) were used with 100 ng cDNA.

Protein isolation of cells and tissue lysates. Cells were harvested and lysed in RIPA buffer (50 mM Tris- $\mathrm{HCl} \mathrm{pH} \mathrm{7.4,} 150 \mathrm{mM} \mathrm{NaCl}, 1 \%$ [v/v] NP-40, 1\% [w/v] sodium deoxycholate, 0.1\% [w/v] SDS, 1 mM EDTA) and complete protease inhibitor (Roche).

Tissue lysates were prepared with the Ultra-Turrax T8 tissue homogenizer (IKA-WERKE) in RIPA buffer. After sonication, homogenates were spun down at $16,900 \mathrm{~g}$ to remove nuclei and insoluble debris. The supernatant was stored at $-80^{\circ} \mathrm{C}$ until further use.

Western blot. Proteins were denatured at $90^{\circ} \mathrm{C}$ for 5 minutes in Laemmli buffer. After separation by SDS-PAGE, proteins were transferred onto PVDF membranes (Whatman). Membranes were blocked in 1\% BSA and incubated with primary antibodies at appropriate dilutions overnight at $4^{\circ} \mathrm{C}$. The following primary antibodies were used: rabbit anti-GMPPA (Proteintech) 1:500, rb anti-GMPPB (Abcam, ab125421) 1:500, rabbit anti-GAPDH (Santa Cruz Biotechnology, sc-25778) 1:1,000, rabbit anti-Myc (MilliporeSigma, 06-549) 1:1,000, rabbit anti-FLAG M2 (Sigma-Aldrich, F7425) 1:1,000, mouse anti-MBP (Sigma-Aldrich, M6295) 1:4,000, mouse anti-GST (gift of Jürgen Wienands, Institute of Cellular and Molecular Immunology at the University Medical Center Göttingen, Germany), rabbit anti-laminin (Abcam, ab11575) 1:500, rabbit anti-nidogen (Abcam, ab14511) 1:500, rabbit anti-collagen IV (Abcam, ab6586) 1:500, self-made antibodies mouse anti-oligomannose and mouse anti-paucimannose 1:50 (gift from Rüdiger Horstkorte, Institute for Physiological Chemistry, Martin-Luther-University Halle-Wittenberg, Halle, Germany), mouse anti- $\alpha$-DG IIH6C4 (MilliporeSigma, 05-593) 1:250, mouse anti- $\alpha$-DG VIA4 (MilliporeSigma, 05-298) 1:250, sheep anti-core$\alpha-D G$ (R\&D Systems) 1:1,000, mouse anti-ubiquitin (Thermo Fisher Scientific, 13-1600) 1:1,000, 1:500, rabbit anti-P-ERK 1/2 (Cell Signaling Technology, 4370) 1:4,000, and rabbit anti-ERK 1/2 (Cell Signaling Technology, 4695) 1:4,000. Primary antibodies were 


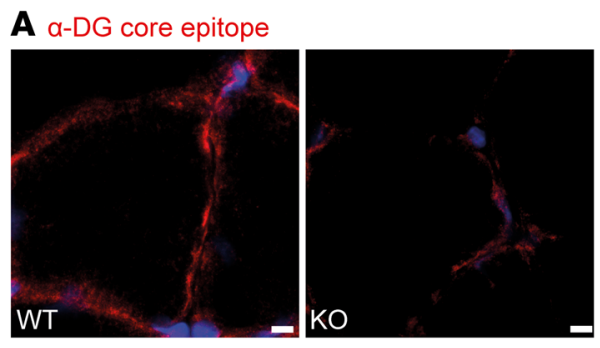

B $\beta$-DG

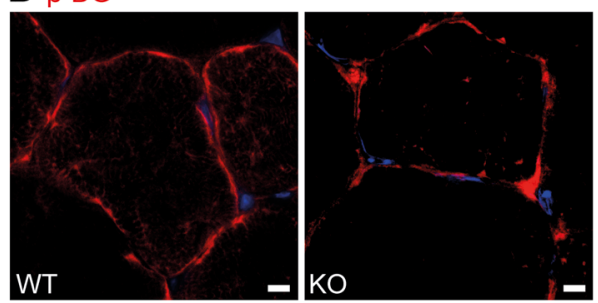

D $\alpha-D G ॥ H 6 C 4$

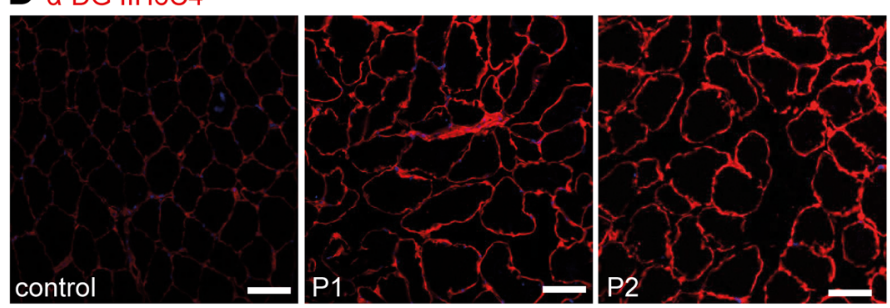

E $a-D G$ VIA4

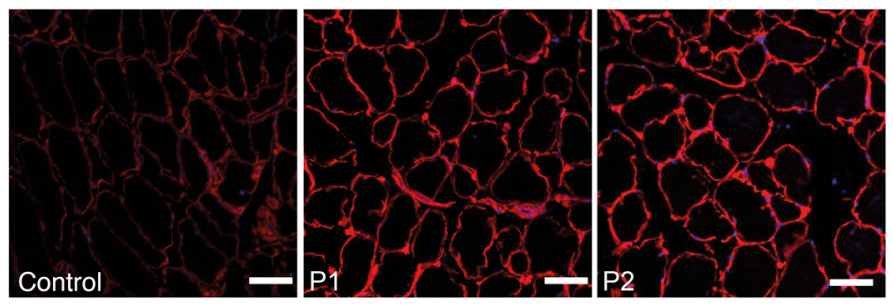

F $a$-DG core

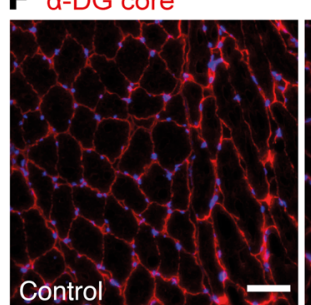

Control

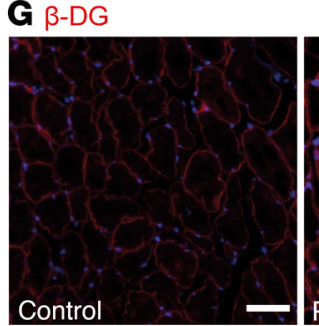

H Laminin
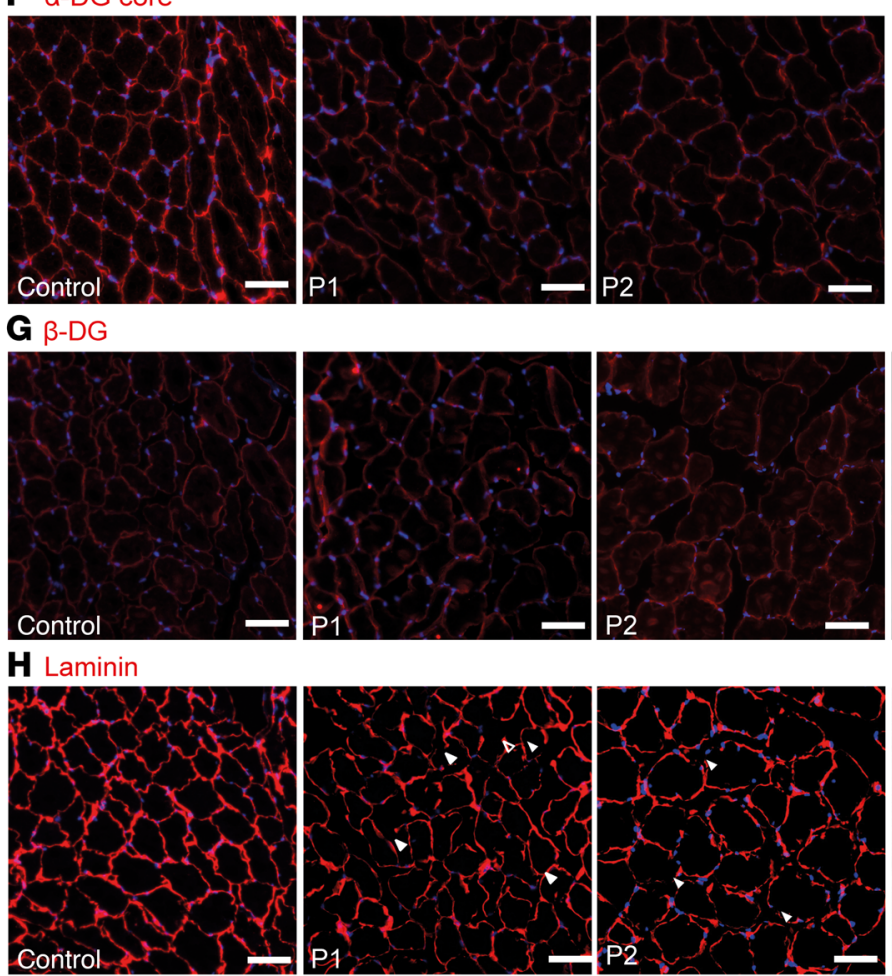

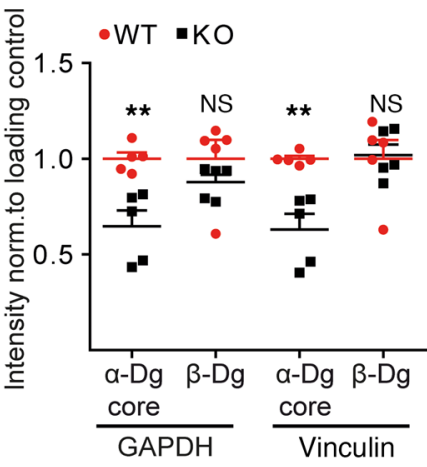

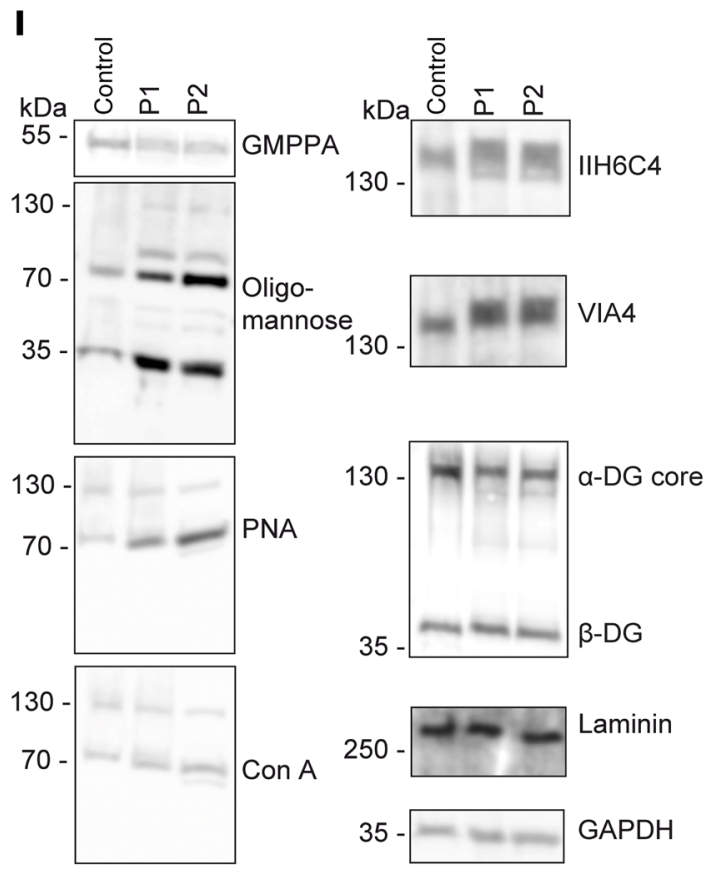

J $\alpha$-Actinin
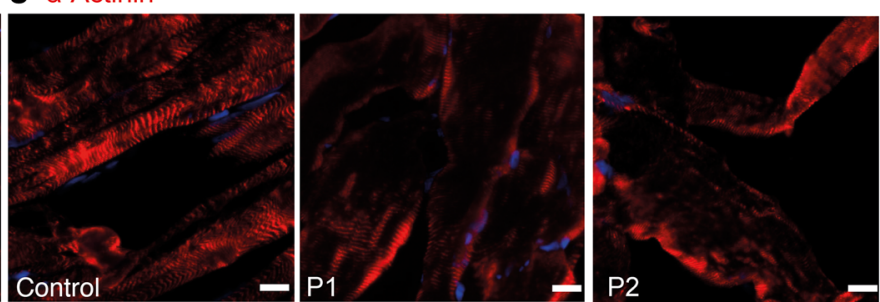

K
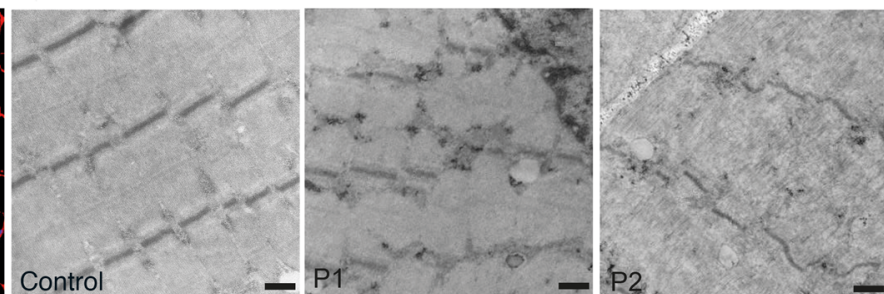
Figure 7. Hyperglycosylation and decreased abundance of $\alpha$-DC in KO mice and in patients with AAMR syndrome. (A) Signals for the $\alpha$-DC core were decreased in skeletal muscle sections of Gmppa-KO mice at 12 months of age. Overviews and control staining after deglycosylation with PNGase $\mathrm{F}$ are shown in Supplemental Figure 5. (B) $\beta$-DC signals were unchanged. Scale bars (A and B): $5 \mu \mathrm{m}$. (C) Skeletal muscle $\alpha$-DC abundance was decreased in 12-month-old KO mice; $\beta$-DC abundance was unchanged ( $n=5$ per group; 2-way ANOVA with Bonferroni post hoc test). Vinculin and CAPDH served as loading control. The quantification after enzymatic deglycosylation is shown in Supplemental Figure 5C. (D and E) Immunostaining of cross-sections of musculus quadriceps femoris biopsies from patients (P1, P2) for the glycosylation-specific $\alpha$-DG epitopes. (D) IIH6C4 and (E) VIA4 showed increased signal intensities compared with the control. (F) Signal intensities for the $\alpha$-DG core protein were decreased in patients. (G) $\beta$-DC signal intensities were unchanged. (H) In patients, laminin signals appear irregular (arrowheads). Scale bars (D-H): $50 \mu \mathrm{m}$. (I) Immunoblot analyses of skeletal muscle lysates showed decreased band intensities for GMPPA and the $\alpha$-DG core in patient samples; signals for oligomannose, PNA, and the glycosylation-specific $\alpha$-DG epitope were increased. Note the shift of the bands for $\alpha-D G$ to a larger size in patients. The signals for Con A, $\beta-D G$, and laminin were unchanged. CAPDH served as loading control. Additional loading controls are displayed in Supplemental Figure 7. (J) The regular $\alpha$-actinin staining pattern was almost absent in patient samples. Scale bars: $10 \mu \mathrm{m}$. (K) The ultrastructural analyses revealed disarranged myofibers and irregular Z-discs in patients. Scale bars: $500 \mathrm{~nm}$. Quantitative data are presented as mean \pm SEM with individual data points. ${ }^{* *} P<0.005$.

detected with HRP-conjugated secondary antibodies. To detect glycans, we used the following lectins: biotin-PNA (Vector Labs) and biotin-Con A (gift from Christian Thiel, Group Congenital Disorders of Glycosylation, University Hospital Heidelberg, Germany), which were detected with HRP-conjugated streptavidin. Detection was performed with the SuperSignal Western Blot Enhancer Kit (Thermo Fisher Scientific). The quantification of bands was done with ImageJ. All blots were repeated at least once.

Coimmunoprecipitation and GST pulldown. HEK-293T cells (ATCC) were either transfected with human cDNAs encoding GMPPAMyc, GMPPB-FLAG, GMPPA-T334P-Myc, GMPPA-N182D-Myc, FLAG-GMPPA, GMPPB-FLAG plasmids, and/or Myc-tagged cDNA constructs missing either the C- (GMPPA aa1-205) or N-terminal part (GMPPA aa206-420) using the Lipofectamine 2000 reagent (Invitrogen). After 24 hours, cells were lysed (20 mM imidazole pH 8.0, 150 $\mathrm{mM} \mathrm{NaCl}, 2 \mathrm{mM} \mathrm{MgCl}$, $300 \mathrm{mM}$ sucrose, 0.25\% [v/v] Triton X-100) and centrifuged at $16,900 \mathrm{~g}$. The supernatant was incubated with either Myc- or FLAG-coupled agarose beads overnight at $4^{\circ} \mathrm{C}$. The beads were washed and boiled at $90^{\circ} \mathrm{C}$ for 10 minutes in Laemmli sample buffer.

Recombinant human GST-GMPPB and MBP-GMPPA proteins were generated in E. coli (ATCC) using the pGEX4T1 and pMal-c2X or pMAL-c5X plasmids and purified on glutathione-agarose (SigmaAldrich) or amylose-resin (New England Biolabs). Comparable amounts of protein were preincubated in lysis buffer $(20 \mathrm{mM}$ imidazole pH 8.0, $150 \mathrm{mM} \mathrm{NaCl}, 2 \mathrm{mM} \mathrm{MgCl}_{2}, 300 \mathrm{mM}$ sucrose, $0.25 \%$ [v/v] Triton X-100) and centrifuged at $16,900 \mathrm{~g}$. The supernatant was incubated with GSH-agarose beads for 45 minutes at $4^{\circ} \mathrm{C}$ and washed, and protein complexes were eluted with Laemmli buffer by boiling at $90^{\circ} \mathrm{C}$ for 5 minutes. After separation of proteins by SDS-PAGE, Western blot analyses were performed with anti-MBP and anti-GST antibodies.

Histology and immunohistochemistry. Muscle tissue was cryosectioned into $5 \mu \mathrm{m}$ thick sections. For histological analyses, sections were stained with H\&E (Sigma-Aldrich). Images were captured with a Zeiss AxioLab A1 microscope and further analyzed by ImageJ.

For the NADH diaphorase staining, cryo-sections were incubated for 30 minutes at $37^{\circ} \mathrm{C}$ in NADH solution $(8 \mathrm{mg} \mathrm{NADH} / 5 \mathrm{~mL} 0.05 \mathrm{M}$ Tris buffer, $\mathrm{pH}$ 7.6) and nitro-blue tetrazolium (NBT) solution (10 $\mathrm{mg} \mathrm{NBT} / 5 \mathrm{~mL}, 0.05 \mathrm{M}$ Tris buffer, $\mathrm{pH}$ 7.6) at a 1:1 ratio. Afterwards, sections were washed and excessive NBT was removed with acetone. Images were captured with a Zeiss AxioLab A1 microscope and further analyzed by ImageJ.

For Picro-Sirius red staining analyses, sections were stained with hematoxylin (Sigma-Aldrich). After washing, sections were stained in $0.5 \%$ (w/v) Picro-Sirius red solution (Sigma-Aldrich) for 1 hour and then washed in acidified water. Images were captured with a petrographic microscope (Axio Imager Z.2, Zeiss) and further analyzed by ImageJ. For TUNEL staining, cryosections were processed according to the manufacturer's instructions (Roche, 11684795910).

Brain tissue was cryo-sectioned into $12 \mu \mathrm{m}$ thick sections. Immunofluorescence staining was performed in Shandon chambers (Thermo Fisher Scientific). Sections were fixed with $4 \%$ PFA and rinsed in PBS; $0.25 \%$ (v/v) Triton X-100 in $1 \times$ PBS was used to permeabilize cells. After blocking, the following primary antibodies were applied overnight at $4^{\circ} \mathrm{C}$ : rabbit anti-laminin (Abcam, ab11575) 1:200, rabbit anti-nidogen (Abcam, ab14511) 1:200, rabbit anti-collagen IV (Abcam, ab6586) 1:100, mouse anti-oligomannose and anti-paucimannose (Rüdiger Horstkorte) both 1:50, mouse anti- $\alpha$-DG IIH6C4 (MilliporeSigma, 05-593) 1:100, mouse anti- $\alpha$-DG VIA4 (MilliporeSigma, 05-298) 1:100, goat anti- $\alpha$-DG (Abcam, ab136665) 1:100, rabbit anti- $\beta$-DG (GeneTex, GTX124225) 1:100, mouse anti- $\alpha$-actinin (EA-53, Abcam, ab9465) 1:200, mouse anti-myosin heavy chain IIB (BF-F3, DSHB) undiluted, mouse anti-myosin heavy chain IIA (sc-71, DSHB) undiluted, mouse anti-myosin heavy chain 7 (A4.840, DSHB) undiluted, mouse antiNeuN (MilliporeSigma, MAB377) 1:250, and rabbit anti-calbindin (D28-K, Swant, CB38) 1:250. To detect glycans, we used biotin PNA (Vector Labs) and biotin Con A (gift from Christian Thiel) in a dilution of 1:50. Corresponding secondary antibodies were obtained from Invitrogen. Nuclei were stained with DAPI 1:10,000 (Invitrogen). Sections were mounted with Fluoromount-G (Southern Biotech). Images were taken with a Zeiss LSM880 Airyscan confocal microscope. Z-projections with average intensities processed with ImageJ are shown. The acquisition parameters and image processing were identical.

Deglycosylation. For the analysis of deglycosylated proteins by Western blot, proteins were digested using an enzymatic Carborelease kit (QA Bio) containing PNGase F (Chryseobacterium meningosepticum), $\mathrm{O}$-glycosidase (Streptococcus pneumoniae), neuraminidase (Arthrobacter ureafaciens), $\beta$-galactosidase (Streptococcus pneumoniae), $\beta$-N-acetylglucosaminidase (Streptococcus pneumonia) as well as $\alpha$-mannosidase (Jack Bean, QA Bio) were used following the manufacturer's instructions for denaturing conditions. Afterward, immunoblotting was performed as described above.

For immunostaining of deglycosylated sections, cryo-tissue sections were incubated with $0.3 \%(\mathrm{w} / \mathrm{v})$ SDS and $60 \mathrm{mM}$ DTT for 10 minutes at $65^{\circ} \mathrm{C}$, followed by washing with PBS and treatment with $10 \mathrm{U} / \mu \mathrm{L}$ PNGase $\mathrm{F}$ (Promega) in $50 \mathrm{mM} \mathrm{Na}_{2} \mathrm{HPO}_{4}$ supplemented with $1 \%(\mathrm{v} / \mathrm{v}) \mathrm{NP}-40$ at $37^{\circ} \mathrm{C}$ for 1.5 hours. Afterward, immunofluorescence staining was performed as already described.

PLAs. PLAs were performed with the Duolink in situ red starter kit mouse/rabbit (DUO92101) according to the manufacturer's instruc- 

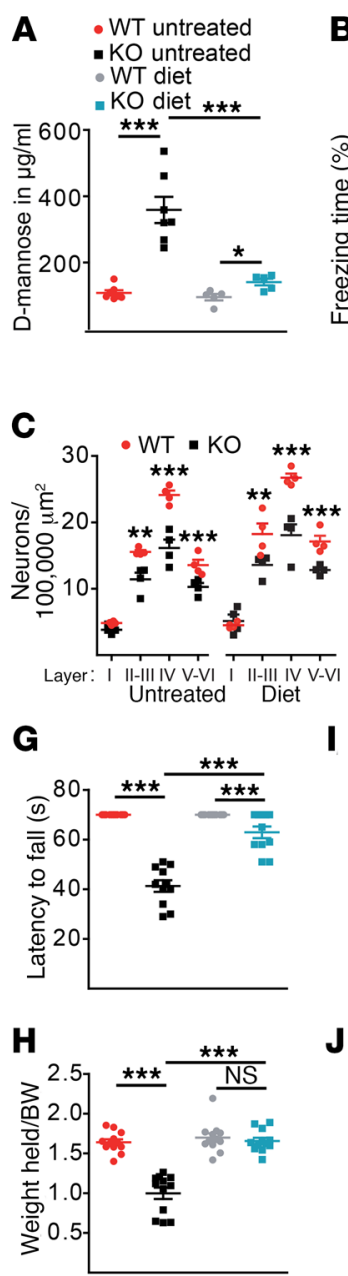
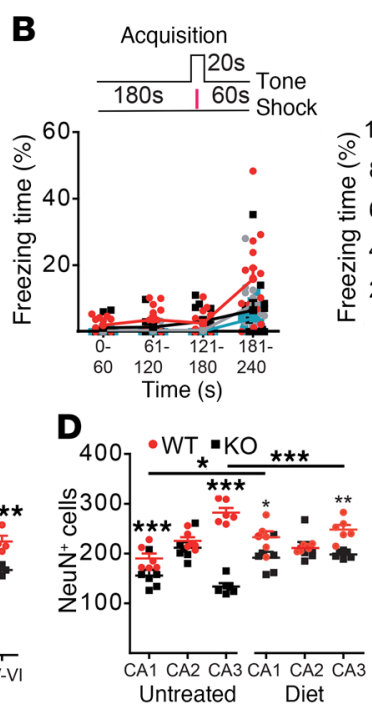
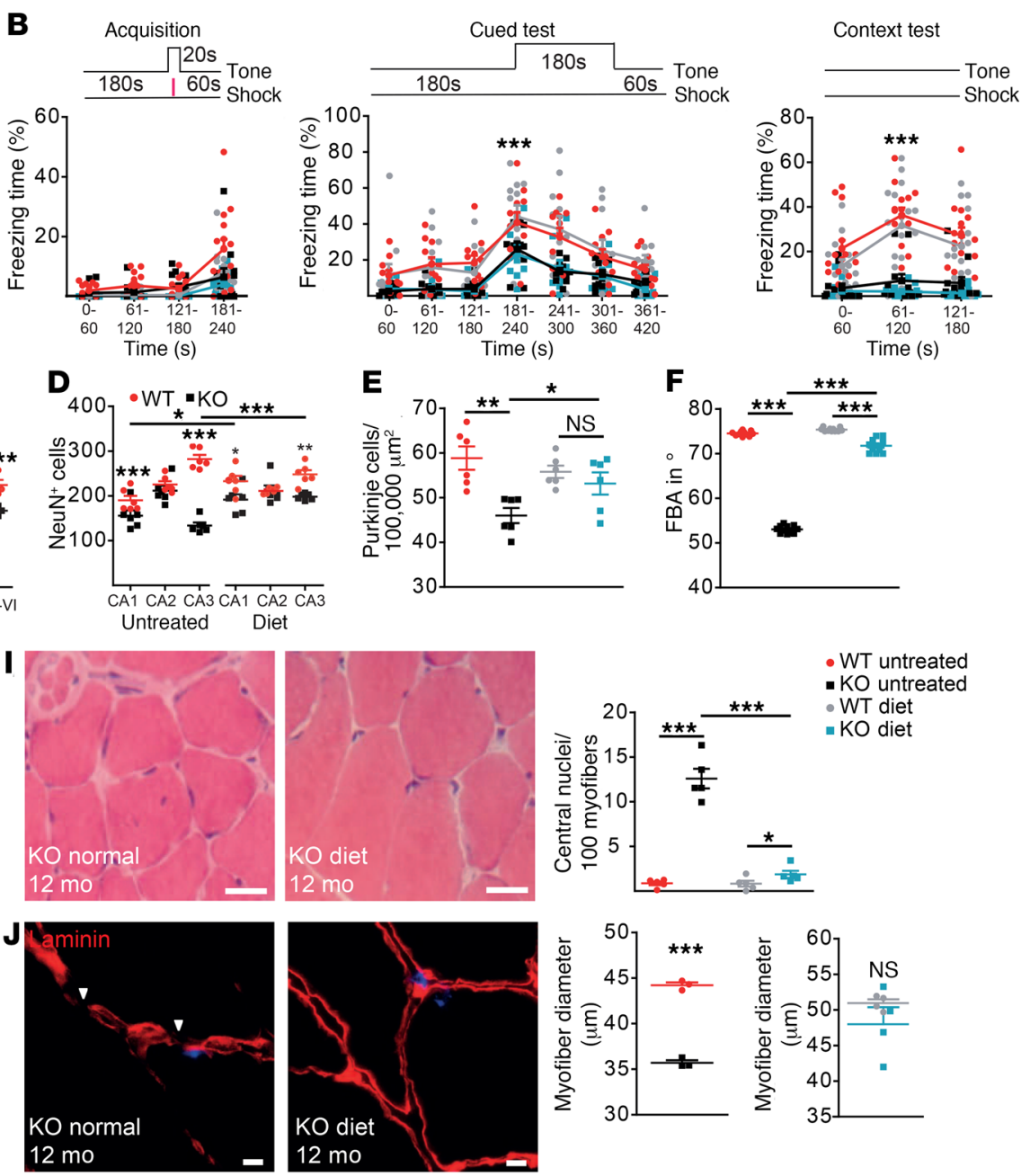

$\mathbf{K}$
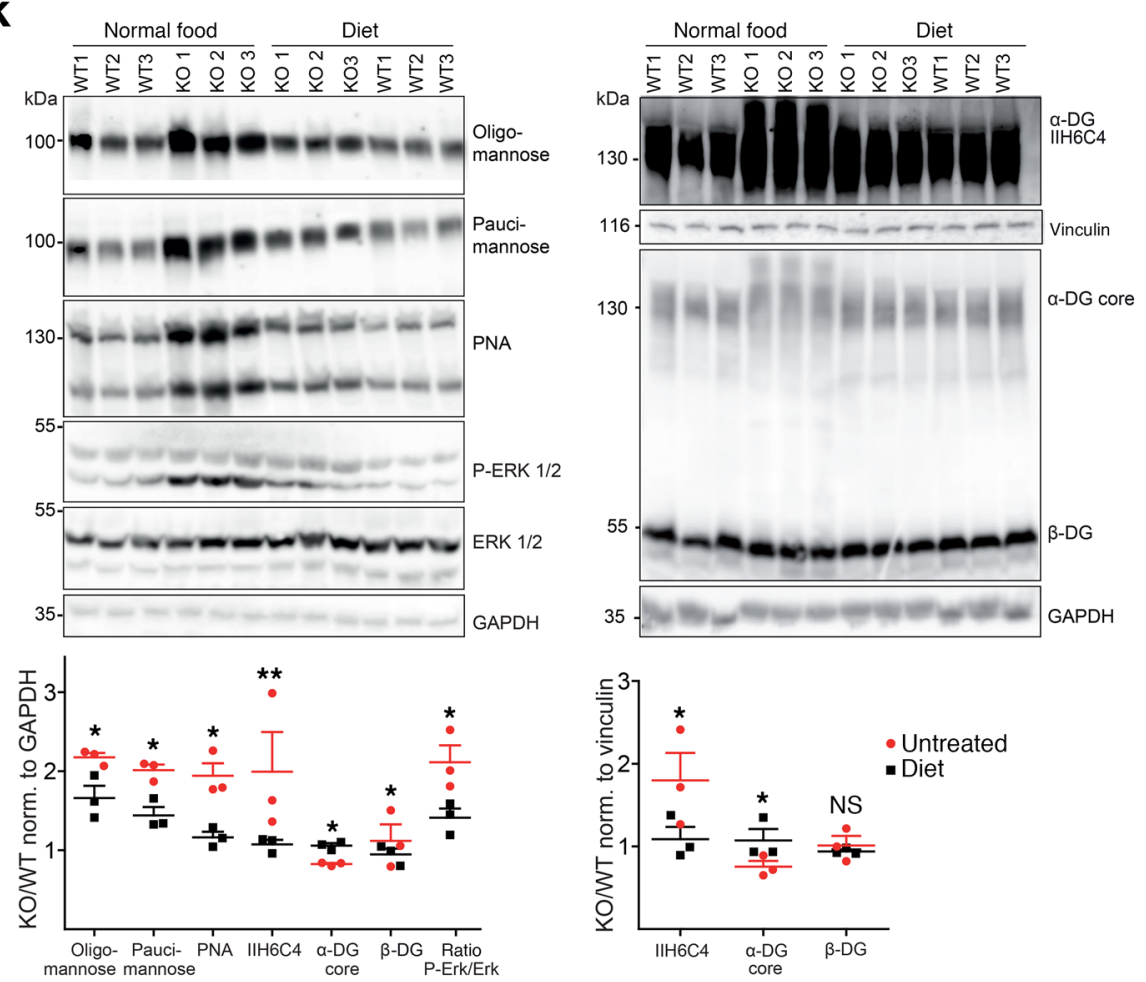
Figure 8. Motor impairments of $\mathrm{Gmppa-KO}$ mice are largely prevented by dietary mannose depletion. (A) Serum mannose almost normalizes upon dietary mannose depletion ( $n=5-7$ per group; 2-way ANOVA, Bonferroni post hoc test). (B) No improvement in the fear-conditioning paradigm of 3-month-old KO mice on a mannose-depleted diet starting at P14 ( $n=12$ per group; 2-way ANOVA, Bonferroni post hoc test). (C) Dietary intervention did not prevent abnormal cortical layering ( $n=4$ per group; 2-way ANOVA, Bonferroni post hoc test). Additional loading controls are displayed in Supplemental Figure 7. (D and E) Hippocampal neuron loss (D) and Purkinje cell loss (E) was attenuated by mannose restriction ( $n=$ 6 per group; 2-way ANOVA, Bonferroni post hoc test). (F) The foot-base angle (FBA) did not deteriorate in mannose-restricted KO mice ( $n=11$ per group; 2-way ANOVA, Bonferroni post hoc test). (C) Almost normal latency to fall off an inverted screen in treated $\mathrm{KO}$ mice ( $n=11$ per group; 2-way ANOVA, Bonferroni post hoc test). (H) The maximal weight held with forepaws did not deteriorate in mannose-depleted KO mice ( $n=12$ mice per group; 2-way ANOVA, Bonferroni post hoc test). (I) Dietary intervention normalized skeletal muscle morphology. $\mathrm{H} \& \mathrm{E}$-stained sections of 12-month-old mice and quantification of centralized nuclei ( $n=12$ mice per group; 2-way ANOVA, Bonferroni post hoc test). Scale bars: $50 \mu \mathrm{m}$. (J) Treatment normalized laminin distribution and myofiber diameter in $\mathrm{KO}$ mice ( $n=12$ mice per group; unpaired 2-tailed Student's $t$ test). Scale bars: $5 \mu \mathrm{m}$. (K) Immunoblot analyses of skeletal muscle lysates (white column: ratio WT/KO untreated mice, black column: ratio WT/KO treated mice; $n=$ 3 per group; 1-way ANOVA, Bonferroni post hoc test). Signals for oligo- and paucimannose, PNA, glycosylation- and core-specific $\alpha$-DG epitope, and P-ERK $1 / 2$ normalized upon treatment. $\beta$-DG and binding immunoglobin protein abundances were unchanged. Vinculin and GAPDH served as loading control. Statistical evaluation compared differences between genotypes in either the treated or untreated cohort or between both cohorts. Quantitative data are presented as mean \pm SEM with individual data points. ${ }^{*} P<0.05 ;{ }^{* *} P<0.005 ;{ }^{* *} P<0.0005$.

tions (Sigma-Aldrich) with rabbit anti-GMPPA (Proteintech) and mouse anti-GMPPB (Novus Biologicals) antibodies in a 1:25 dilution.

Enzyme activity assays. Recombinant human GST-GMPPA, MBP-GMPPA, GST-GMPPB, MBP-GMPPB, and variant proteins were generated in E. coli using the pGEX4T1 and pMal-c2X or pMALc5X plasmids and purified on glutathione-agarose (Sigma-Aldrich) or amylose-resin (New England Biolabs). GDP-mannose-pyrophosphorylase activity of recombinant enzymes was measured by determining the quantity of inorganic phosphate generated from pyrophosphate in the presence of mannose-1-phosphate $(150 \mu \mathrm{M})$, GTP $(300 \mu \mathrm{M})$, and excess pyrophosphatase $(1 \mathrm{U} / \mathrm{mL}$; Merck) in assay buffer $(25 \mathrm{mM}$ Tris$\mathrm{HCl} \mathrm{pH} \mathrm{7.5,} 150 \mathrm{mM} \mathrm{NaCl}, 4 \mathrm{mM} \mathrm{MgCl}_{2}, 0.01 \%$ [v/v] Tween 20,1 mM dithiothreitol in ultrapure water supplemented with protein inhibitor cocktail; Roche) at $37^{\circ} \mathrm{C}$ for 40 minutes as described previously (17). Biochemical reactions were terminated by adding equal volumes of revelation buffer $(0.03 \%$ [w/v] malachite green [Sigma-Aldrich], 0.2\% $[\mathrm{w} / \mathrm{v}]$ ammonium molybdate, $0.05 \%$ [v/v] Triton $\mathrm{X}-100$ in $0.7 \mathrm{M} \mathrm{HCl}$ ) at $30^{\circ} \mathrm{C}$ for 5 minutes and absorbance measured at $650 \mathrm{~nm}$. For measuring GMPPB activity in the presence of GMPPA, $20 \mathrm{ng} / \mu \mathrm{L}$ GMPPB was mixed with $20 \mathrm{ng} / \mu \mathrm{L}$ GMPPA in assay buffer supplemented with pyrophosphatase $1 \mathrm{U} / \mathrm{mL}, 300 \mu \mathrm{M}$ GTP, $150 \mu \mathrm{M}$ mannose-1-phosphate, and increasing GDP-mannose concentrations.

Sugar measurements. Blood was taken from unfasted mice and incubated on ice for 15 minutes. Samples were centrifuged for $10 \mathrm{~min}$ utes at $4^{\circ} \mathrm{C}$ and $4,000 \mathrm{~g}$. Sugars were measured in the supernatant with the D-mannose, D-fructose, D-glucose kit (Megazyme, K-MANGL).

Mass spectrometry analysis of skeletal muscle proteins. Homogenized quadriceps muscle samples were lysed in RIPA buffer followed by reduction, alkylation, and subsequent acetone precipitation. Protein pellets were digested into peptides, labeled with 10plex Tandem Mass Tags (Thermo Fisher Scientific), and fractionated by high $\mathrm{pH}$ reverse-phase chromatography, as described earlier (40). The resulting fractions were combined into 24 pools and analyzed in an Orbitrap Fusion Lumos Tribrid Mass Spectrometer (Thermo Fisher Scientific) using a synchronous precursor selection (SPS) /MS3 method (41). Raw data were processed using Proteome Discoverer v2.0 (Thermo Fisher Scientific) and searched against a Uniprot mouse database using Mascot v2.5.1 (Matrix Science). Differential expression analysis was performed using limma (42). The mass spectrometry proteomics data were deposited to the ProteomeXchange Consortium via the PRIDE (43) partner repository with the dataset identifier PXD014260. Gene set enrichment analysis (GSEA) was performed using WebGestalt (44).

Glycome analysis of skeletal muscle. Mice were fasted for 24 hours. Protein lysis was performed in lysis buffer ( $50 \mathrm{mM}$ Tris- $\mathrm{HCl}, 100 \mathrm{mM}$ $\mathrm{NaCl}, 1 \mathrm{mM}$ EDTA, and proteinase inhibitor cocktail). Samples were sonicated and then centrifuged at $4,000 \mathrm{~g}$ at $4^{\circ} \mathrm{C}$ and the supernatant recovered. For $\mathrm{N}$-glycan and $\mathrm{O}$-glycan analysis, $200 \mu \mathrm{g}$ proteins were dissolved in $\mathrm{PB} \mathrm{pH} 6.5$ (250 $\mathrm{mM} \mathrm{NaH}_{2} \mathrm{PO}_{4}, 250 \mathrm{mM} \mathrm{Na}_{2} \mathrm{HPO}_{4}$ ) and SDS was added to a final concentration of $1 \%(\mathrm{w} / \mathrm{v})$. Proteins were denatured at $95^{\circ} \mathrm{C}$ for 5 minutes. The buffer was diluted to $160 \mathrm{mM}$ with water and Ipegal at a final concentration of $1 \%(\mathrm{w} / \mathrm{v})$. Next, $\mathrm{N}$ glycan release was performed using $1 \mathrm{U}$ of PNGase F (N-Zyme Scientifics). $\mathrm{N}$-glycans were subsequently cleaned up, permethylated, and measured by MALDI-TOF mass spectrometry as described earlier (45). $\mathrm{O}$-glycans were released with $\mathrm{NaOH} / \mathrm{NaBH}_{4}$ and purified over self-made $\mathrm{C} 18 /$ Dowex $\mathrm{H}^{+}$columns followed by a methanolic desalting step in the vacuum centrifuge and a permethylation reaction. The spectra were acquired in $m / z 300-2000$ region in the positive ion mode $[\mathrm{M}+\mathrm{Na}]^{+}$. For every spectrum acquisition, 10,000 shots were collected. The spectra were acquired at $100 \mathrm{~Hz}$ frequency. Detector gain was set up to $1638 \mathrm{~V}$ and the analog offset was $51 \mathrm{mV}$.

Experiments using primary myoblasts. Primary myoblasts isolated from WT mice were seeded in growth medium (F10, 20\% [v/v] FBS, $2 \%$ penicillin/streptomycin, $2.5 \mathrm{ng} / \mathrm{mL}$ basic fibroblast growth factor (bFGF); all from Gibco) on collagen-coated culture dishes. The next day, cells were treated with differentiation medium (DMEM, SigmaAldrich; $5 \%$ [v/v] horse serum, Gibco; $2 \%$ penicillin/streptomycin, Gib$\mathrm{co}$ ) and allowed to differentiate for 2 days. Then, cells were transfected with siRNAs against either control (siScr), dystroglycan (siDAG1, Dharmacon), or GMPPA (siGMPPA, Novus Biologicals) according to the manufacturer's protocol using Lipofectamine RNAiMax (Invitrogen). After 4 days of differentiation, cells were fixed with 2\% PFA, permeabilized, blocked, and stained with antibodies directed against myogenin (F5D, DSHB) 1:2 and myosin heavy chain (MF20, DSHB) 1:2 overnight at $4^{\circ} \mathrm{C}$ followed by an incubation with the corresponding secondary antibodies (Invitrogen). Nuclei were stained with DAPI $(10 \mu \mathrm{g} / \mathrm{mL}$, Invitrogen). Images were taken with the Axio Imager and Axio Observer.Z1 (Carl Zeiss) and further analyzed with the Zen software (Carl Zeiss).

For CHX experiments, primary myoblasts were seeded, differentiated, and transfected as described above. After 3 days, cells were treated with $8 \mu \mathrm{g} / \mathrm{mL}$ CHX for 24 hours. Then cells were harvested with RIPA buffer. After sonication, homogenates were spun down at $16,900 \mathrm{~g}$ to remove nuclei and insoluble debris. The supernatant was stored at $-80^{\circ} \mathrm{C}$.

Experiments using C2C12 myoblasts. C2C12 cells (ATCC) were seeded in growth medium (DMEM, Sigma-Aldrich; 10\% [v/v] FBS, 
Gibco; 2\% [v/v] penicillin/streptomycin; Gibco). The next day, cells were treated with differentiation medium (DMEM, Sigma-Aldrich; $2 \%$ horse serum, Gibco) and allowed to differentiate. Transfection with siRNAs against either control (siScr), dystroglycan (siDag1), or GMPPA (siGMPPA) was carried out according to the manufacturer's protocol using Lipofectamine RNAiMax (Invitrogen). Fixation and immunostaining were performed as described above.

GDP-mannose measurements. Mice were euthanized and organs were dissected and immediately frozen in liquid nitrogen. Organ homogenates were run on HPLC with a modified gradient to enhance separation of NDP sugars as described previously (7).

Electron microscopy. The human biopsies were immersed in $2.5 \%$ (v/v) glutaraldehyde (EMS, 16210) followed by 2 washes in cacodylate buffer (EMS, 21300) at $\mathrm{pH} 7.2$ and $0.15 \mathrm{M}$ and postfixation done in $1 \%$ (v/v) osmium tetraoxide (EMS, 19100), followed by 2 washes in cacodylate buffer and tissue dehydrated in the following progressive eth$\mathrm{yl}$ alcohol concentrations at 30\%, 50\%, 70\%, 80\%, 96\%, and $100 \%$ (v/v). For an adequate infiltration, muscle samples were changed to propylene oxide (EMS, 20401). The infiltration was carried out with epoxy resin. The procedure described was carried out in a Leica EM TP automatic processor. Processed biopsies were cut in $75 \mathrm{~nm}$ thick sections and mounted on 200 mesh copper grids. The contrast was attained with 5\% (w/v) uranyl acetate (EM Grade) and lead citrate with Reynold's method, and the samples analyzed in a transmission electron microscope FEI model Tecnai BioTwin at $80 \mathrm{kV}$.

Mice were perfused transcardially with $4 \%$ PFA and $2.5 \%$ glutaraldehyde in PBS. Sciatic nerve was removed and postfixed. Afterwards, nerves were washed with $0.1 \mathrm{M}$ cacodylate buffer $\mathrm{pH} 7.3$ and postfixed in $0.1 \mathrm{M}$ cacodylate buffer. Tissue was dehydrated in the following progressive acetone concentrations at 30\%, 50\%, 70\%, 90\%, $95 \%$, and $100 \%(\mathrm{v} / \mathrm{v})$. For contrast, $1 \%(\mathrm{w} / \mathrm{v})$ uranyl acetate was added to $50 \%(\mathrm{v} / \mathrm{v})$ acetone. The infiltration was carried out with epoxy resin. Processed biopsies were cut in $50 \mathrm{~nm}$ thick sections (Reichert Ultracut S, Leica) and analyzed in a transmission electron microscope JEM 1400 (JEOL) at $80 \mathrm{kV}$.

Statistics. For statistical analysis, raw data were analyzed for normal distribution with the Kolmogorov-Smirnov test or with graphical analysis using the box-plot and QQ-plot. If appropriate, we either used 1-way ANOVA, 2-way ANOVA, or Student's $t$ test. $P$ values less than 0.05 were considered significant. For all data, means with SEM are shown.

Study approval. All animal experiments were approved by the TLLV. For tests with samples of patients with AAMR syndrome, who underwent muscle biopsy as part of the diagnostic workup because of progressive muscle weakness, written informed consent was obtained stating that part of the specimen could be used for research purposes. Control samples were obtained from the Telethon Biobank.

Data and software availability. Mass spectrometry datasets and MALDI-TOF spectra of glycans are included in Supplemental Table 1.

\section{Author contributions}

PF, HH, MJJ, SCS, SM, KB, LL, IK, TK, JM, BM, TH, AKH, JCH, SG, JVM, and LG performed experiments and analyzed data. PF, $\mathrm{CK}, \mathrm{AO}, \mathrm{OH}, \mathrm{VB}, \mathrm{JVM}$, and CAH wrote the paper. PF, RH, JW, TM, $\mathrm{CK}, \mathrm{OMM}, \mathrm{AO}, \mathrm{OH}, \mathrm{VB}, \mathrm{JVM}$, and CAH interpreted data and contributed to the conceptual design of the study. $\mathrm{CAH}$ initiated and coordinated the study.

\section{Acknowledgments}

This study was funded by the DFG GRK 2155 ProMoAge to CAH, $\mathrm{OH}, \mathrm{RH}, \mathrm{CK}$, and AO and DFG MA-3975/2-1 to JVM. We thank Emile van Schaftingen and Guido Bommer for continuous support. We thank Katrin Schorr, Manuela Neumann, and Johanna Fischer for excellent technical support; Katrin Buder and the Histology and Electron Microscopy Facility of the Fritz Lipmann Institute (FLI) for electron microscopy analysis; and Joanna M. Kirkpatrick and the Mass Spectrometry Core Facility of the FLI for support with mass spectrometry. This study is supported by the EUROGLYCAN-omics network, E-Rare-3 Joint Transnational Call. The FLI is a member of the Leibniz Association and is financially supported by the Federal Government of Germany and the State of Thuringia.

Address correspondence to: Christian A. Hübner, University Hospital Jena, Am Klinikum 1, Jena 07747, Germany. Phone 0049.3641.9396800; Email: christian.huebner@med.uni-jena.de
1. Jaeken J. Congenital disorders of glycosylation (CDG): it's (nearly) all in it! J Inherited Metab Dis. 2011;34(4):853-858.

2. Belaya K, et al. Mutations in GMPPB cause congenital myasthenic syndrome and bridge myasthenic disorders with dystroglycanopathies. Brain. 2015;138(pt 9):2493-2504.

3. Cabrera-Serrano M, et al. Expanding the phenotype of GMPPB mutations. Brain. 2015;138 (pt 4):836-844.

4. Carss KJ, et al. Mutations in GDP-mannose pyrophosphorylase $\mathrm{B}$ cause congenital and limb-girdle muscular dystrophies associated with hypoglycosylation of $\alpha$-dystroglycan. Am J Hum Genet. 2013;93(1):29-41.

5. Jensen BS, et al. GMPPB-associated dystroglycanopathy: emerging common variants with phenotype correlation. Hum Mutat. 2015;36(12):1159-1163.

6. Ning B, Elbein AD. Cloning, expression and characterization of the pig liver GDP-man- nose pyrophosphorylase. Evidence that GDP-mannose and GDP-Glc pyrophosphorylases are different proteins. Eur J Biochem. 2000;267(23):6866-6874.

7. Koehler K, et al. Mutations in GMPPA cause a glycosylation disorder characterized by intellectual disability and autonomic dysfunction. Am J Hum Genet. 2013;93(4):727-734.

8. Ervasti JM, Campbell KP. Membrane organization of the dystrophin-glycoprotein complex. Cell. 1991;66(6):1121-1131.

9. Ibraghimov-Beskrovnaya O, et al. Primary structure of dystrophin-associated glycoproteins linking dystrophin to the extracellular matrix. Nature. 1992;355(6362):696-702.

10. Dumont NA, et al. Dystrophin expression in mus cle stem cells regulates their polarity and asymmetric division. Nat Med. 2015;21(12):1455-1463.

11. Barresi R, Campbell KP. Dystroglycan: from biosynthesis to pathogenesis of human disease. JCell Sci. 2006;119(pt 2):199-207.
12. Rodriguez CI, et al. High-efficiency deleter mice show that FLPe is an alternative to Cre-loxP. Nat Genet. 2000;25(2):139-140.

13. Schwenk F, et al. A cre-transgenic mouse strain for the ubiquitous deletion of loxP-flanked gene segments including deletion in germ cells. Nucleic Acids Res. 1995;23(24):5080-5081.

14. Beetz C, et al. A spastic paraplegia mouse model reveals REEP1-dependent ER shaping. J Clin Invest. 2013;123(10):4273-4282.

15. Phillips RG, LeDoux JE. Differential contribution of amygdala and hippocampus to cued and contextual fear conditioning. Behav Neurosci. 1992;106(2):274-285.

16. Jin $X$, et al. Crystal structure of potato tuber ADP-glucose pyrophosphorylase. EMBOJ. 2005;24(4):694-704.

17. Mao W, et al. Biochemical analysis of leishmanial and human GDP-mannose pyrophosphorylases and selection of inhibitors as new leads. Sci Rep. 2017;7(1):751. 
18. Endo T. Aberrant glycosylation of alpha-dystroglycan and congenital muscular dystrophies. Acta Myol. 2005;24(2):64-69.

19. Martin PT. Dystroglycan glycosylation and its role in matrix binding in skeletal muscle. Glycobiology. 2003;13(8):55R-66R.

20. Cohn RD, et al. Disruption of DAG1 in differentiated skeletal muscle reveals a role for dystroglycan in muscle regeneration. Cell. 2002;110(5):639-648.

21. Benitez EO, et al. A novel GMPPA mutation in two adult sisters with achalasia, alacrima, short stature, dysmorphism, and intellectual disability. Mol Syndromol. 2018;9(2):110-114.

22. Sharma V, Freeze HH. Mannose efflux from the cells: a potential source of mannose in blood. J Biol Chem. 2011;286(12):10193-10200.

23. Sivaraman J, et al. Crystal structure of Escherichia coli glucose-1-phosphate thymidylyltransferase (RffH) complexed with dTTP and Mg2+.JBiol Chem. 2002;277(46):44214-44219.

24. Jensen SO, Reeves PR. Domain organisation in phosphomannose isomerases (types I and II). Biochim Biophys Acta. 1998;1382(1):5-7.

25. Szumilo T, et al. GDP-mannose pyrophosphorylase. Purification to homogeneity, properties, and utilization to prepare photoaffinity analogs. J Biol Chem. 1993;268(24):17943-17950.

26. Raetz CR, Roderick SL. A left-handed parallel beta helix in the structure of UDP-N-acetylglucosamine acyltransferase. Science. 1995;270(5238):997-1000.

27. Campbell KP. Three muscular dystrophies: loss of cytoskeleton-extracellular matrix linkage. Cell. 1995;80(5):675-679.

28. Inamori K, et al. Dystroglycan function requires xylosyl- and glucuronyltransferase activities of LARGE. Science. 2012;335(6064):93-96.

29. Yoshida-Moriguchi T, et al. O-mannosyl phosphorylation of alpha-dystroglycan is required for laminin binding. Science. 2010;327(5961):88-92.

30. Longman C, et al. Mutations in the human LARGE gene cause MDC1D, a novel form of congenital muscular dystrophy with severe mental retardation and abnormal glycosylation of alpha-dystroglycan. Hum Mol Genet. 2003;12(21):2853-2861.

31. Brockington $M$, et al. Transgenic overexpression of LARGE induces $\alpha$-dystroglycan hyperglycosylation in skeletal and cardiac muscle. PLoS One. 2010;5(12):e14434.

32. Whitmore $\mathrm{C}$, et al. The transgenic expression of LARGE exacerbates the muscle phenotype of dystroglycanopathy mice. Hum Mol Genet. 2014;23(7):1842-1855.

33. Matthijs G, et al. Mutations in PMM2, a phosphomannomutase gene on chromosome 16p13, in carbohydrate-deficient glycoprotein type I syndrome (Jaeken syndrome). Nat Genet. 1997;16(1):88-92.

34. Niehues R, et al. Carbohydrate-deficient glycoprotein syndrome type Ib. Phosphomannose isomerase deficiency and mannose therapy. JClin Invest. 1998;101(7):1414-1420.

35. Sharma V, et al. Mannose metabolism: more than meets the eye. Biochem Biophys Res Commun.
2014;453(2):220-228.

36. Alton $\mathrm{G}$, et al. Direct utilization of mannose for mammalian glycoprotein biosynthesis. Glycobiology. 1998;8(3):285-295.

37. Schneider A, et al. Successful prenatal mannose treatment for congenital disorder of glycosylation-Ia in mice. Nat Med. 2012;18(1):71-73.

38. Dehay C, Kennedy H. Cell-cycle control and cortical development. Nat Rev Neurosci. 2007;8(6):438-450.

39. Deacon RM. Measuring the strength of mice. J Vis Exp. 2013;(76):2610.

40. Heinze I, et al. Species comparison of liver proteomes reveals links to naked mole-rat longevity and human aging. BMC Biol. 2018;16(1):82.

41. Muntel J, et al. Comparison of protein quantification in a complex background by DIA and TMT workflows with fixed instrument time. J Proteome Res. 2019;18(3):1340-1351.

42. Ritchie ME, et al. limma powers differential expression analyses for RNA-sequencing and microarray studies. Nucleic Acids Res. 2015;43(7):e47.

43. Perez-Riverol Y, et al. The PRIDE database and related tools and resources in 2019: improving support for quantification data. Nucleic Acids Res. 2019;47(D1):D442-D450.

44. Liao Y, et al. WebGestalt 2019: gene set analysis toolkit with revamped UIs and APIs. Nucleic Acids Res. 2019;47(W1):W199-W205.

45. Biskup K, et al. Serum glycome profiling: a biomarker for diagnosis of ovarian cancer. J Proteome Res. 2013;12(9):4056-4063. 